\title{
Dissipative versus reversible contributions to macroscopic dynamics: the role of time-reversal symmetry and entropy production
}

\author{
Helmut R. Brand ${ }^{1,2} \cdot$ Harald Pleiner ${ }^{1} \cdot$ Daniel Svenšek ${ }^{3}$ \\ Received: 15 February 2018 / Revised: 11 September 2018 / Accepted: 13 September 2018 / Published online: 5 October 2018 \\ (C) The Author(s) 2018
}

\begin{abstract}
We discuss the time-reversal behavior of dynamic cross-couplings among various hydrodynamic degrees of freedom in liquid crystal systems. Using a standard hydrodynamic description including linear irreversible thermodynamics, we show that the distinct thermodynamic requirements for reversible and irreversible couplings lead to experimentally accessible differences. We critically compare our descriptions with those of existing standard continuum mechanics theories, where time-reversal symmetry is not adequately invoked. The motivation comes from recent experimental progress allowing to discriminate between the hydrodynamic description and the continuum mechanics approach. This concerns the dynamics of Lehmanntype effects in chiral liquid crystals and the dynamic magneto-electric response in ferronematics and ferromagnetic nematics, a liquid multiferroic system. In addition, we discuss the consequences of time-reversal symmetry for flow alignment of the director in nematics (or pretransitional nematic domains) and for the dynamic thermo-mechanical and electro-mechanical couplings in textured nematic liquid crystals.
\end{abstract}

Keywords Macroscopic dynamics $\cdot$ Lehmann effect $\cdot$ Flow alignment $\cdot$ Thermo-mechanical effects ·

Ferromagnetic nematics

\section{Introduction}

Time-reversal symmetry is one of the fundamental properties of microscopic as well as of macroscopic systems. For the latter, the second law of thermodynamics states that a process in a thermally isolated system is reversible if and only if the entropy of the system remains unchanged during this process. In the field of complex fluids, liquid crystals for instance, the seminal work, which is rigorous in this respect, is by Martin et al. (1972). Nevertheless, for nematic

Harald Pleiner

pleiner@mpip-mainz.mpg.de

Helmut R. Brand

brand@uni-bayreuth.de

Daniel Svenšek

daniel.svensek@fmf.uni-lj.si

1 Max Planck Institute for Polymer Research, 55021 Mainz, Germany

2 Theoretische Physik III, Universität Bayreuth, 95440 Bayreuth, Germany

3 Department of Physics, Faculty of Mathematics and Physics, University of Ljubljana, 1000 Ljubljana, Slovenia liquid crystals in particular, there existed a description based on continuum mechanics that did not use time-reversal symmetry (Ericksen 1960, 1961; Leslie 1968). Basically, this approach was adapted by de Gennes (1975) giving the impression that both descriptions are equivalent. Due to its mechanical picture, the Leslie-Ericksen picture is still used mostly in the experimental community, while on the theoretical side both approaches are used in parallel, e.g., for the description of the dynamics of biaxial nematics (Brand and Pleiner 1981; Liu 1981; Saupe 1981).

However, with the advent of ever more complex liquid crystal and soft matter systems, like bent-core nematic and smectic liquid crystals, systems involving tetrahedral order, polar and magnetic liquids, polymers, and elastomers, ordered active matter, it has become obvious that a thorough theoretical description based on thermodynamics is inevitable. Time-reversal symmetry is an important element of that. In addition, despite the common belief, there are subtle differences between the two schools of describing the dynamics, even for simple liquid crystal phases including uniaxial nematics and cholesterics. Carefully designed experiments are needed, and nowadays available, to identify these theoretically decisive, yet practically often rather small differences in real 
complex fluid systems. Therefore, it is important for the scientific field to understand and recognize the approaches that firmly reside on thermodynamic grounds, and tell them apart from other less stringent yet possibly almost equally successful models.

There is a very general formalism to derive non-equilibrium reversible and irreversible equations, GENERIC (Grmela and Öttinger 1997; Öttinger and Grmela 1997), and Grmela (2002), which is very similar in spirit to the hydrodynamic method used in this paper: There are two functionals, energy and entropy, that govern the statics and the irreversible dynamics, respectively, with the fundamental laws of thermodynamics built in. The GENERIC equations are set up in terms of Poisson brackets and irreversible ('Grmela') brackets making use of Onsager symmetry and Casimir skewsymmetry (Öttinger 2014). The hydrodynamic description is somewhat simpler, since it is confined to non-equilibrium situations that are close to equilibrium. Nevertheless, equations derived in that regime, like the Navier-Stokes equations for simple fluids, can subsequently be used to successfully describe situations far from equilibrium, like chaos or turbulence. Hydrodynamic equations can be set up without relying on any microscopic pictures or models, although there are connections to linear response theory (cf. Appendix B). The strongest argument for using the hydrodynamic method is that not only thermodynamics is taken care of (by means of time-reversal symmetry), but also the spatial symmetries are lucidly dealt with. This is of great advantage for the description of nematic and nematiclike systems. In particular, for some conditions, imposed by thermodynamic requirements, the spatial structure of the coupling between different variables is crucial. We are not aware of any derivation of macroscopic equations for nematic-type systems using GENERIC, but we are pretty sure that they would be compatible with the hydrodynamic ones we are using.

To derive macroscopic equations, other approaches have been suggested. They include, for example, the use of hierarchies of orientational correlation functions (Doi 1981; Kuzuu and Doi 1983). However, This approach has been shown (Pleiner et al. 2002) to be incompatible with the systematically derived macroscopic equations for the nematic order parameter $Q_{i j}$.

We use a stringent hydrodynamic description based on (linear irreversible) thermodynamics considering the symmetries and broken symmetries involved. We focus on macroscopic consequences of time-reversal behavior in the hydrodynamic regime, describing the low frequency, long-wavelength response. Of particular interest are dynamic cross-couplings among the various degrees of freedom. Here, different time-reversal behavior discriminates reversible from irreversible couplings, with zero entropy production in the former case and positive entropy production in the latter. This is the fundamental notion of the hydrodynamic description and is discussed in detail in the "Thermodynamic forces and currents in linear irreversible thermodynamics" section. Failure of dealing correctly with time-reversal symmetry, generally results in a violation of irreversible thermodynamics. We show how the form of dynamic cross-couplings is different depending on whether they are of reversible or irreversible nature. This will be the key feature of the subsequent discussion of the various dynamic effects under consideration.

In the "Lehmann-type effects in chiral liquid crystals" section, we discuss how time-reversal symmetry influences the nature and the structure of dynamic cross-coupling terms describing Lehmann-type effects in chiral liquid crystals (Madhusudana and Pratibha 1987; Brand and Pleiner 1988; Madhusudana and Pratibha 1989; Tabe and Yokoyama 2003; Svenšek et al. 2006, 2008; Oswald and Dequidt 2008; Pleiner and Brand 2010; Seki et al. 2011; Brand et al. 2013; Yoshioka et al. 2014; Yamamoto et al. 2015) and where a recent experiment (Sato et al. 2017) paves the way to corroborate this description. We compare hydrodynamics with various continuum mechanics approaches. We will show that all continuum mechanics approaches have missed the so-called static Lehmann-type couplings. The dynamic Lehmann couplings were rewritten by de Gennes (1975) (subsequently taken over by Oswald and Pieranski (2005) with the erroneous claim that they follow from the reversible part of the dynamics, disregarding the correct time-reversal symmetry behavior of the quantities involved. Instead, those couplings are irreversible (with a nonzero entropy production) and that leads to differences that can experimentally be detected when the inverse Lehmann effects are considered.

The "Reversible dynamic coupling of flow to the nematic director dynamics" section is devoted to dynamic crosscouplings between flow and an orientational degree of freedom, in particular, the director in nematic liquid crystals and pretransitional nematic domains in the isotropic phase above the nematic one. The flow alignment in external shear flow is governed by a dynamic parameter that is part of the reversible dynamics. This has been overlooked by Leslie (1968II) and de Gennes (1975) who have taken these effects as dissipative viscosities. As a result, erroneous positivity conditions are claimed that correlate dissipative with reversible parameters in violation of linear irreversible thermodynamics.

In the "Dynamic thermo-mechanical effects in textured nematic liquid crystals" section, it is shown, using timereversal behavior, that the dynamic thermo-mechanical and electro-mechanical effects in textured nematic liquid crystals are reversible. On the contrary, Akopyan and 
Zeldovich (1984) have erroneously assumed these thermomechanical couplings to be dissipative. The failure of their description becomes apparent when both, the direct and the inverse effects are measured simultaneously.

In the "Thermodynamic forces and currents in ferronematics and ferromagnetic nematics" section, we discuss as another example, the coupled dynamics of the magnetization and the director field in ferronematics and ferromagnetic nematics (Jarkova et al. 2001, 2003). The different behavior under time reversal of the nematic director and of the magnetization leads to characteristic, experimentally detectable features (Potisk and et al. 2017, 2018). Those features have not been found using a continuum mechanics approach (Raikher and Stepanov 1996, 1999), where the director and the magnetization are treated on equal footing, disregarding their different time-reversal behavior.

Similar discussions of an erroneous use of time reversal of dynamic cross-coupling terms in bio-inspired active systems, regarding active stresses (Brand et al. 2014) and active transport (Pleiner et al. 2016II) have been given previously.

In Appendix A, we list the complete set of hydrodynamic nematic equations as a reference and in Appendix B, we recall the impact of time-reversal symmetry on memory and frequency matrices in the projector formalism of Forster, Mori, and Zwanzig in the hydrodynamic regime.

\section{Thermodynamic forces and currents in linear irreversible thermodynamics}

In this section, we summarise briefly the essence of linear irreversible thermodynamics, in particular, we elucidate the role of time-reversal symmetry, as this will be one of the major tools used throughout the manuscript. A more explicit exposition for nematic liquid crystals is provided in Appendix A as reference. Although this material is not new, we will make use of it in the main part of this manuscript.

We describe systems that are in local thermodynamic equilibrium, that is, all microscopic variables are in equilibrium averaged over a suitably chosen volume and time interval. Then only a few slow variables, the state variables $x_{\alpha}$, which are space-time fields, describe the macroscopic dynamic behavior of the system. The first law of thermodynamics requires that the state variables are related due to energy conservation by the Gibbs relation (de Groot and Mazur 1962; Martin et al. 1972; Pleiner and Brand 1996)

$T d \sigma=d \varepsilon-F_{\alpha} d x_{\alpha}$

connecting the macroscopic variables to the entropy density $\sigma$, with $T$ the temperature and $\varepsilon$ the energy density. The index $\alpha$ denotes the appropriate variables involved and
Einstein summation is assumed. The fields $F_{\alpha}$ are thermodynamically conjugate to the variables. They are defined as partial derivatives of an energy functional. Deviations of $F_{\alpha}$ from their equilibrium values are the thermodynamic forces.

Since the time-reversal symmetry is crucial, we will discriminate, where appropriate, between variables that are even under time reversal $x_{\alpha}^{e}(t)=+x_{\alpha}^{e}(-t)$, or $\epsilon^{T}\left(x_{\alpha}^{e}\right)=$ +1 , and the odd ones $x_{\alpha}^{o}(t)=-x_{\alpha}^{o}(-t)$ with $\epsilon^{T}\left(x_{\alpha}^{o}\right)=-1$. The former comprise, e.g., mass density $\rho$, concentration $c$, and the nematic director $n_{i}$, while in the latter group one finds the momentum density $g_{i}$, the angular momentum $\omega_{i}$, and the magnetization $m_{i}$. Temperature, energy density, and entropy density are time-reversal symmetric, with the result that the thermodynamically conjugates must have the same time-reversal behavior as the appropriate variables $x_{\alpha}$ and Eq. 1 could be written as $T d \sigma=d \varepsilon-F_{\alpha}^{e} d x_{\alpha}^{e}-F_{\alpha}^{o} d x_{\alpha}^{o}$.

The macroscopic variables satisfy dynamic balance equations of the general form

$\dot{x}_{\alpha}+J_{\alpha}\left(\left\{F_{\alpha}\right\}\right)=0$

with the dot denoting a partial time derivative. The currents $J_{i}$ are associated with the dynamics of all other macroscopic variables, generated by the thermodynamic forces. For the conserved degrees of freedom, like momentum density or mass density, the (thermodynamic) currents $J_{\alpha}=\nabla_{i} j_{i}^{\alpha}$ have the form of a divergence of a (physical) current, reflecting the local conservation law $\dot{x}_{\alpha}+\nabla_{i} j_{i}^{\alpha}=0$ for those variables. In the liquid crystal literature, sometimes the currents of non-conserved variables are called "quasi-currents," and only in the case of conserved variables "current" is used. Generally, this distinction is not really necessary.

Non-conserved variables enter macroscopic dynamics in two variants, either they are symmetry variables representing Goldstone modes connected to spontaneously broken continuous symmetries, or they are slowly varying mesoscopic variables. Examples for the former are the nematic director $n_{i}$, the smectic phase variable describing rotations of a preferred direction and translation of layers, respectively. For the latter type of non-conserved macroscopic variables, a discussion of the specific system is necessary-an example is relative rotations in a nematic elastomer. We will make the distinction between conserved and non-conserved quantities only where necessary.

Of course, also the energy density obeys a local conservation law

$$
\dot{\varepsilon}+\nabla_{i} j_{i}^{\varepsilon}=0
$$

while the entropy density is only conserved for reversible processes, but not for irreversible ones

$$
\dot{\sigma}+\nabla_{i} j_{i}^{\sigma}=2 R / T
$$


It is this place where the second law of thermodynamics enters macroscopic dynamics: The dissipation function vanishes, $R=0$, for reversible processes, while it is positive, $R>0$, for irreversible ones. This distinction is unique (and complete), since on the macroscopic level the dynamic processes are either time-reversal symmetric or change sign under time reversal, which exactly corresponds to the distinction, reversible or irreversible.

In the following, we discuss the implication of the second law of thermodynamics on the form of the currents, $J_{\alpha}$. To facilitate this, the currents are (uniquely) separated

$J_{\alpha}=J_{\alpha}^{R}+J_{\alpha}^{D}$

into a reversible (R) and an irreversible part (D) according to their time-reversal behavior. The reversible part $J_{\alpha}^{R}$ transforms like $\dot{x}_{\alpha}$, while the irreversible one, $J_{\alpha}^{D}$ has the opposite behavior and therefore transforms like $x_{\alpha}$. Explicitly, the time-reversed form of Eq. 2, $T_{R}\left[\dot{x}_{\alpha}+J_{\alpha}^{R}+J_{\alpha}^{D}=0\right]$ reads $\dot{x}_{\alpha}+J_{\alpha}^{R}-J_{\alpha}^{D}=0$.

The distinction (5) defines the time-reversal behavior of $J_{\alpha}^{R}$ and $J_{\alpha}^{D}$ relative to that of $\dot{x}_{\alpha}$. To get the absolute timereversal behavior one has to take into account, whether the variable itself is even or odd. Writing $\dot{x}_{\alpha}^{e}+J_{\alpha}^{e R}+J_{\alpha}^{e D}=0$ one finds $J_{\alpha}^{e R}$ to be odd and $J_{\alpha}^{e D}$ to be even, while for $\dot{x}_{\alpha}^{o}+J_{\alpha}^{o R}+J_{\alpha}^{o D}=0$ one gets $J_{\alpha}^{o R}$ to be even and $J_{\alpha}^{o D}$ to be odd under time reversal

$\epsilon^{T}\left(J_{\alpha}^{e R}\right)=-1$ and $\epsilon^{T}\left(J_{\alpha}^{e D}\right)=+1$

$\epsilon^{T}\left(J_{\alpha}^{o R}\right)=+1$ and $\epsilon^{T}\left(J_{\alpha}^{o D}\right)=-1$

These results apply identically to the (physical) currents, $j_{i}^{\alpha}$. The source term in Eq. 4 is an example of $-J_{\alpha}^{D}$ in Eq. 5, since it is nonzero only for dissipative processes. Because the variable $\sigma$ is even, it behaves as $J_{\alpha}^{e D}$ and therefore, the dissipation $R$ has to be even under time reversal. Physically that means, a dissipative process produces the same amount of entropy, whether time runs forward or backward, or more pronounced, a process that increases entropy does not decrease it, when the time axis is inverted.

Putting all dynamic equations into the Gibbs relation (1) we get

$$
\begin{array}{r}
\quad-j_{i}^{\sigma R} \nabla_{i} T+F_{\alpha} J_{\alpha}^{R}-j_{i}^{\alpha R} \nabla_{i} F_{\alpha}=0 \\
2 R=-j_{i}^{\sigma D} \nabla_{i} T+F_{\alpha} J_{\alpha}^{D}-j_{i}^{\alpha D} \nabla_{i} F_{\alpha}>0
\end{array}
$$

as a compact form of the second law of thermodynamics. For conserved quantities $J_{\alpha}=\nabla_{i} j_{i}^{\alpha}$, for both, the reversible and the irreversible case, and the appropriate contributions to the second law of thermodynamics show that in this case the actual force is $\nabla_{i} F_{\alpha}$, rather than $F_{\alpha}$. To get this form, surface contributions like $\nabla_{i}\left(j_{i}^{\alpha} F_{\alpha}\right)$ have been subsumed into the divergence of the energy current density $\nabla_{i} j_{i}^{\varepsilon}$. This procedure will be discussed in detail explicitly when we present the macroscopic dynamics of nematic liquid crystals in Appendix A. The basic meaning of Eqs. 7 and 8 is that all dissipative contributions have to add up to something positive, while the reversible processes cancel each other to zero entropy production.

To be more specific, we employ linear irreversible thermodynamics, a systematic framework to derive macroscopic equations for a set of macroscopic variables $\left\{x_{\alpha}\right\}$ for situations that are driven out of equilibrium by a set of thermodynamic forces $\left\{F_{\alpha}\right\}$ (de Groot and Mazur 1962; Landau and Lifshitz 1959; Martin et al. 1972; Forster 1975). Here, the relations between the currents induced by the thermodynamic forces are linear, $J_{\alpha}=\zeta_{\alpha \beta} F_{\beta}$. This does not mean the resulting dynamic equations are linear, since the dynamic coupling matrices $\zeta_{\alpha \beta}$ are generally functions of the state variables $\left\{x_{\alpha}\right\}$ and of possible external fields. Only if the $\zeta_{\alpha \beta}$ are constant, they produce linear contributions to the dynamic equations.

To discuss the linear current/force relation in more detail, we make the distinction between reversible and irreversible processes

$$
\begin{aligned}
& J_{\alpha}^{R}=\zeta_{\alpha \beta}^{R} F_{\beta} \\
& J_{\alpha}^{D}=\zeta_{\alpha \beta}^{D} F_{\beta}
\end{aligned}
$$

introducing reversible and irreversible transport parameters, $\zeta_{\alpha \beta}^{R}$ and $\zeta_{\alpha \beta}^{D}$, respectively. It should be noted that the qualifiers "reversible" and "irreversible" refer to the macroscopic processes they describe, not to their microscopic origin, cf. the discussion after Eq. 15 and Appendix B.

We have already shown that the forces $F_{\alpha}$ have the same time-reversal signature as the appropriate variables, $\epsilon_{\alpha}^{T}=+1$ or -1 for even and odd variables, respectively. The same is true for the dissipative currents $J_{\alpha}^{D}$, while the reversible ones, $J_{\alpha}^{R}$, have the opposite time reversible signature $-\epsilon_{\alpha}^{T}$. As a result, the dynamic coupling matrices $\zeta_{\alpha \beta}$ must transform as

$\epsilon^{T}\left(\zeta_{\alpha \beta}^{R}\right)=-\epsilon_{\alpha}^{T} \epsilon_{\beta}^{T}$
$\epsilon^{T}\left(\zeta_{\alpha \beta}^{D}\right)=+\epsilon_{\alpha}^{T} \epsilon_{\beta}^{T}$

In the linear case, where the coupling matrices are constants, one can conclude that dissipative couplings are only possible among variables of the same time-reversal behavior (including self-couplings), while reversible couplings requires opposite time-reversal behavior. Examples for the dissipative case are the Soret/Dufour effect, Eqs. 90 and 91 (and Newtonian viscosity, Eq. 89), while nematic flow alignment and backflow, Eqs. 82 and 83, represents a reversible coupling. In the nonlinear case, there is the possibility that the coefficients $\zeta_{\alpha \beta}$ depend on a quantity that is odd under time reversal, like the magnetization or a magnetic field. Then, also dissipative (reversible) couplings 
are possible among variables of opposite (equal) timereversal symmetry. Examples are discussed in Eqs. 53-55 and Eqs. 58-60, respectively.

The linear current-force relation, Eq. 10, allows the dissipation function to be written as, $2 R=\zeta_{\alpha \beta}^{D} F_{\alpha} F_{\beta}$, and to serve as a quadratic potential for deriving dissipative currents by partial derivation according to Eq. 8. It also facilitates the discussion of the symmetry requirements, $\zeta_{\alpha \beta}^{D}=\zeta_{\beta \alpha}^{D}$ and the positivity $R>0$. Taking as an example $R=\frac{1}{2} \zeta_{11}^{D} F_{1}^{2}+\zeta_{12}^{D} F_{1} F_{2}+\frac{1}{2} \zeta_{22}^{D} F_{2}^{2}$, with $F_{1}$ and $F_{2}$ of the same time-reversal behavior, the appropriate currents are

$J_{1}^{D}=\zeta_{11}^{D} F_{1}+\zeta_{12}^{D} F_{2}$

$J_{2}^{D}=\zeta_{22}^{D} F_{2}+\zeta_{21}^{D} F_{1}$

where, according to Eq. 12, the dissipative transport coefficients have to be even under time reversal, (e.g., being constant), irrespective of the (common) time-reversal behavior of the two variables involved. By the very construction of $R$, they fulfill $\zeta_{12}^{D}=\zeta_{21}^{D}$ (Onsager relation), and the conditions $\zeta_{11}^{D}>0, \zeta_{22}^{D}>0, \zeta_{11}^{D} \zeta_{22}^{D}>\left(\zeta_{12}^{D}\right)^{2}$ must hold to guarantee positivity of the entropy production for irreversible processes (8).

If the two degrees of freedom of this example are conserved, one gets equivalent results, when $J_{1,2}^{D}$ is replaced by $-j_{1,2}^{D}$ and $F_{1,2}$ by $\nabla_{i} F_{1,2}$, respectively. An example of that case is the coupled diffusion of heat and concentration, the anisotropic version of which is used in Eqs. 88, 90, and 91. Of course, also dissipative couplings between a conserved variable $\left(F_{2}\right)$ and a non-conserved one $\left(F_{1}\right)$ are possible, but only in systems that lack spatial inversion symmetry, as can be seen from the coupling term in the dissipation function $R=\frac{1}{2} \zeta_{11}^{D} F_{1}^{2}+\zeta_{12, i}^{D} F_{1} \nabla_{i} F_{2}+$ $\frac{1}{2} \zeta_{22}^{D}\left(\nabla_{i} F_{2}\right)^{2}$ (in the isotropic case): $\zeta_{12, i}^{D}$ has to be odd under spatial inversion. An example for that situation is described in cholesteric liquid crystals, Eqs. 17, 18, and 24.

There is no macroscopic potential for reversible currents. Here, one has to write down possible cross-couplings allowed by time-reversal symmetry, Eq. 11, and then use (7) to guarantee zero dissipation $R=0$. If there are reversible cross-couplings between two variables of opposite timereversal behavior

$$
\begin{aligned}
& J_{3}^{R}=\zeta_{34}^{R} F_{4} \\
& J_{4}^{R}=\zeta_{43}^{R} F_{3}
\end{aligned}
$$

the reversible transport coefficients $\zeta_{34}^{R}$ and $\zeta_{43}^{R}$ must be time-reversal symmetric according to Eq. 11, e.g., being constant. Zero entropy production, Eq. 7, requires an antisymmetric relation $\zeta_{34}^{R}=-\zeta_{43}^{R}$, and there is no restriction on the sign or the size of the reversible transport coefficient. In isotropic, simple liquids such a reversible coupling is not possible. Generally, in anisotropic systems, the reversible coupling coefficients come in the form of tensors and the actual condition for zero entropy production depends also on the structure of those tensors. Typical physical examples are flow alignment and backflow in nematic liquid crystals, Eqs. 82 and 83 and the dynamic reversible cross-coupling between the director and the magnetization in ferronematics, Eqs. 54 and 55.

The macroscopic reversible transport coefficients can have two different microscopic origins, cf. Appendix B, either the reactive instantaneous response, or the imaginary part of the (non-instantaneous) memory function. With respect to the latter part, the antisymmetry $\zeta_{34}^{R}=-\zeta_{43}^{R}$ is sometimes called Casimir relation (Öttinger 2014). For macroscopic equations, there is no need to discriminate between the two origins and the antisymmetry relation simply follows from the macroscopic time-reversal behavior (11).

The convective nonlinearities in macroscopic equations, e.g., $J_{\alpha} \sim v_{i} \nabla_{i} x_{\alpha}$, are reversible and describe the transport part of the convective time derivatives (related to Galilei invariance) and the co-rotational part (related to the proper rotational behavior) of, e.g., the nematic director. They are generic and do not come with any phenomenological, material-dependent parameters. Taken all together, they do not increase entropy and fulfill (7) automatically, when the (isotropic) pressure $p$ is expressed as a quadratic function of the variables according to the Gibbs-Duhem relation (Pleiner and Brand 1996). For nematic liquid crystals, this is discussed in more detail in Appendix A, after (74).

To obtain a closed set of equations one needs relations between the variables and the thermodynamic conjugates as defined in Eq. 1. This can be achieved by taking the variational derivative of a (static) potential, typically an energy expression that is linear or nonlinear in the variables (Pleiner and Brand 1996)

$F_{\alpha}=\delta \varepsilon\left(\left\{x_{\alpha}\right\}\right) / \delta x_{\alpha}$

This energy functional has to be a scalar (rotational invariant) and even under time reversal.

\section{Lehmann-type effects in chiral liquid crystals}

More than 100 years ago, Lehmann (1900) observed the rotation of the director in a cholesteric droplet in an external temperature gradient. Only about seven decades later this observation was taken up in modeling by Leslie (1968, 1969) and in a different form by de Gennes (1975). Leslie described Lehmann-type effects using a continuum mechanics description of Ericksen and assumed the Lehmann effect to be due to a chiral dynamic coupling (Leslie 1968). Another two decades later Madhusudana and Pratibha $(1987,1989)$ performed experiments on cholesteric droplets in an external electric field in the two-phase region near the isotropic - cholesteric transition. 
Over the last decade, there has been a strongly increasing interest in the study of the Lehmann effect induced by external forces such as temperature gradients and external electric fields. Most of these studies were using cholesteric droplets in the two-phase region near the isotropiccholesteric transition (Oswald and Dequidt 2008; Yoshioka et al. 2014; Yamamoto et al. 2015). There appears to be only one study using freely suspended chiral smectic films (Seki et al. 2011).

Stimulated by the experiments of Madhusudana and Pratibha, Lehmann effects were examined in the framework of hydrodynamics based on linear irreversible thermodynamics (Brand and Pleiner 1988). It turned out that Lehmann effects in chiral liquid crystals can have two different origins. Either chiral cross-couplings in the dissipative currents are combined with achiral static susceptibilities (called "dynamic Lehmann"), or chiral static cross-couplings are combined with achiral dissipative currents ("static Lehmann"). The former case leads to director rotations due to temperature, density, and concentration gradients and electric fields. The latter case, which involves only temperature, concentration, and density gradients but no electric field, has not been considered in the traditional descriptions.

There is always an inverse Lehmann effect, if there is a direct one, due to the reciprocal nature of cross-couplings within linear irreversible thermodynamics. Inverse effects, where concentration, heat, or electric currents can be generated by director rotation, have only recently been discussed in detail (Svenšek et al. 2008). In all cases, Lehmann effects are related to the dissipative dynamics (and not the reversible one), in contrast to what is claimed sometimes (de Gennes 1975; Oswald and Pieranski 2005).

Macroscopic chirality is described by the existence of a pseudoscalar quantity, the chirality parameter $q_{0}$, that is invariant under spatial rotation, but changes sign $\left(q_{0} \rightarrow\right.$ $-q_{0}$ ) under spatial inversion (odd parity). This pseudoscalar is responsible, in isotropic fluids made of chiral molecules, for the optical activity (the rotation of the plane of polarization of linearly polarized light), and in cholesterics for the helical ground state of the director (due to a chiral linear twist term in the free energy), and for the Lehmann effects.

We note that the origin of macroscopic chirality can also be related to geometric chirality (Bouligand 1969, 1972, Livolant and Bouligand 1978, Rey 2010, Murugesan and Rey 2010, Aguilar-Gutierrez and Rey 2014, 2016) and charge chirality (Murugesan and Rey 2010; Rey 2010; Rey and Herrera-Valencia 2012). These features are in particular important for biological systems.

However, the existence of $q_{0}$ is not necessarily related to chiral molecules, since there are phases, which are chiral, although the molecules are achiral (structural chirality). An example is the $\mathrm{C}_{B 2}$ smectic phase, where achiral bent-core molecules (characterized by a director and a perpendicular polarization) are tilted w.r.t. to the smectic layers. The tilt is responsible for the existence of a $q_{0}$, and since both tilt possibilities are equally likely, but describe opposite handedness, such systems are called ambidextrous chiral (Cladis et al. 1999). Another example of such a structural chirality is the $D_{2}$-symmetric phase that occurs, if there is tetrahedral order in an orthorhombic biaxial nematic (Pleiner and Brand 2014). Generally, any phase with at least one preferred direction that shows a $q_{0}$, for whatever reason, exhibits helicoidal ground states and Lehmann-type effects (Brand et al. 2013).

On the other hand, the existence of a helical ground state does not necessarily mean that the phase is chiral. There are achiral phases with a helical ground state, e.g., the $D_{2 d}$-symmetric phase where uniaxial nematic orientation is suitably combined with tetrahedral order (Pleiner and Brand 2016). Helical structures are favoured by an achiral linear gradient term in the free energy, which is possible due to the broken spatial inversion symmetry of that phase ${ }^{1}$. Since leftand right-handed helices are equally likely, this is called ambidextrous helicity. Such a phenomenon can even occur in an optically isotropic system, when tetrahedral order is combined with transient elasticity (Brand and Pleiner 2017).

Describing cholesteric liquid crystals locally (Martin et al. 1972, Lubensky 1972, 1973, Pleiner and Brand 1996), we use the nematic director $\boldsymbol{n}$ as a state variable, despite the helical ground state. We note that usual chiral nematics cholesterics are biaxial locally and that therefore the single director model is an approximation. As discussed in the textbook by de Gennes (1975), this biaxiality is small for usual thermotropic low molecular weight materials (of order $\left(q_{0} a\right)^{2}$, where $a$ is a typical molecular dimension). This situation changes when lyotropic biaxial nematic phases are chiralized. In this case, the observed textures change (Figueiredo Neto et al. 1985; Pleiner and Brand 1985) and the tendency to twist around different axes leads frequently to complex defect structures (Melnick and Saupe 1987; Pleiner and Brand 1990). For biological materials and processes, frequently an intermediate situation of moderate biaxiality arises (Rey 2010; Rey and Herrera-Valencia 2012; Aguilar Gutierrez and Rey 2016).

We start with the description of the dynamic side of the Lehmann effect. Chirality allows in the dissipation function for additional terms (compared to nematics) of the form

$$
R=R_{n}+q_{0} n_{k} \epsilon_{i j k} h_{j}\left(\psi_{\sigma} \nabla_{i} T+\psi_{c} \nabla_{i} \mu_{c}+\psi_{e} E_{i}\right)
$$

where $R_{n}$ is the dissipation function of a nematic, given in Eq. 88. The chiral terms provide dissipative cross-couplings

\footnotetext{
${ }^{1}$ Although the original and the inverted structures are not identical, they can be brought to coincidence by a mere rotation, rendering the phase achiral.
} 
between $h_{i}$, the thermodynamic force related to the nematic degree of freedom, with gradients of the temperature $T$, osmotic pressure $\mu_{c}$, and with an electric field $E_{i}$, which are the thermodynamic forces related to the thermal degree of freedom, to concentration $c$, and to the electric displacement field $D_{i}$, respectively. In a nematic phase, they are forbidden due to the spatial inversion symmetry of that phase.

For the dissipative part of the director current, $Y_{i}^{D}$ (cf. Eqs. 72 and 80), one gets

$$
\begin{aligned}
Y_{i}^{D}= & \frac{\partial R}{\partial h_{i}}=\frac{1}{\gamma_{1}} \delta_{i k}^{\perp} h_{k} \\
& +q_{0} \epsilon_{i j k} n_{j}\left(\psi_{\sigma} \nabla_{k} T+\psi_{c} \nabla_{k} \mu_{c}+\psi_{e} E_{k}\right)
\end{aligned}
$$

with $\delta_{i k}^{\perp}=\delta_{i k}-n_{i} n_{k}$ and where the second line describes the chiral additions to the nematic case (93). All contributions to Eq. 18 are even under time reversal, that is opposite to $\dot{n}_{i}$, and are therefore dissipative. The reversible current $Y_{i}^{R}$ is odd under time reversal, but does not show any chiral addition.

Now we switch to the static aspects of Lehmann effects. Cholesterics are characterized by a linear contribution in the twist part of the gradient free energy

$\varepsilon_{t w}=\frac{1}{2} K_{2}[\boldsymbol{n} \cdot(\nabla \times \boldsymbol{n})]^{2}+q_{0} L_{2} \boldsymbol{n} \cdot(\nabla \times \boldsymbol{n})$

which is minimized by a helical superstructure of the director with an equilibrium helical pitch $q_{h}=-\boldsymbol{n} \cdot(\nabla \times$ n) $\left.\right|_{\text {eq }}=q_{0} L_{2} / K_{2}$. Often, $L_{2}=K_{2}$ is chosen, although there is no a priori reason for this choice (Frank 1958). For deviations from this ground state, the hydrodynamic free energy involving twist reads, cf. Brand and Pleiner (1988) and in a slightly different context Pleiner and Brand (1987),

$$
\begin{aligned}
\varepsilon= & \frac{1}{2} K_{2}\left[\boldsymbol{n} \cdot(\nabla \times \boldsymbol{n})+q_{h}\right]^{2} \\
& -q_{0}\left[\boldsymbol{n} \cdot(\nabla \times \boldsymbol{n})+q_{h}\right]\left(\tau_{c} \delta c+\tau_{\sigma} \delta \sigma+\tau_{\rho} \delta \rho\right)
\end{aligned}
$$

showing chiral (static) cross-couplings between the director degree of freedom and the scalar state variables.

The thermodynamic force ("molecular field") $h_{i}$ follows from the energy by variational derivative, Eqs. 16 and 67. For rigid rotations of the homogeneous helix one gets

$h_{i}^{c h}=q_{0} \epsilon_{i j k} n_{j}\left(\tau_{c} \nabla_{k} c+\tau_{\sigma} \nabla_{k} \sigma+\tau_{\rho} \nabla_{k} \rho\right)$

that only contains the chiral terms. Hence, in the first line of Eq. 18, the result (21) leads to contributions rather similar to those in the second line of Eq. 18. Since in this case, there is no backflow, $Y_{i}^{R}$ of Eq. 83 is zero and the dynamics of $n_{i}$ is given by $Y_{i}^{D}$ only, which describes Lehmann rotations

$n \times \dot{n}=-n \times Y^{D}=\Omega$ in the plane perpendicular to the driving forces. In terms of experimentally viable forces, we get

$$
\begin{aligned}
\boldsymbol{\Omega} & =q_{0}\left(\psi_{\sigma}+\psi_{c} \chi_{1}-\gamma_{1}^{-1} \tau_{\sigma} \chi_{4}\right) \nabla T \\
& +q_{0}\left(\psi_{c} \chi_{2}-\gamma_{1}^{-1}\left[\tau_{\rho}+\tau_{\sigma} \chi_{5}\right]\right) \nabla \rho \\
& +q_{0}\left(\psi_{c} \chi_{3}-\gamma_{1}^{-1}\left[\tau_{c}+\tau_{\sigma} \chi_{6}\right]\right) \nabla c \\
& +q_{0} \psi_{e} \boldsymbol{E}
\end{aligned}
$$

where $\chi_{1 \ldots 6}$ are combinations of various static susceptibilities (Svenšek et al. 2008). The individual frequency contributions are generally composed of parts that are products either ("dynamic Lehmann") of a chiral dissipative transport parameter $\left(\psi_{c, \sigma, e}\right)$, Eq. 17, with achiral static susceptibilities $\chi_{1,2,3}$, or ("static Lehmann") of the achiral dissipative transport parameter $1 / \gamma_{1}$, Eq. 88 , with the chiral static susceptibilities $\left(\tau_{c, \sigma, \rho}\right)$, Eq. 20 , except for an electric field, where the latter case is not possible in linear order of $E$.

It should be noted that in actual experiments the Lehmann frequencies depend on the boundary conditions chosen. For instance, for torque-free boundaries with $\boldsymbol{n} \times$ $\boldsymbol{h}=0$ the helical pitch gets a linear profile, $q_{h}=q_{h 0}+q_{h}^{\prime} z$, and the parts with the chiral static susceptibilities drop out of Eq. 23.

All frequencies (23) are linear in the external driving field and in the pseudoscalar chirality parameter $q_{0}$. They change sign when going from the left-handed version of a substance to its right-handed one. There is the possibility to have a finite Lehmann effect $\left(q_{0} \neq 0\right)$, even for a compensated mixture, where the helical pitch is zero $\left(q_{h} \sim L_{2}=0\right.$ meaning there is no helix) (Pleiner and Brand 2010).

It has been shown experimentally that the existence of a helical structure is not necessary for detecting Lehmann effects: in Langmuir monolayers of chiral smectic systems that do have a $q_{0}$, which results in a helix in three-dimensional samples, Lehmann rotations have been observed by Tabe and Yokoyama (2003), despite the lack of a helix in a monolayer. The results have been shown to be fully compatible (Svenšek et al. 2006) with the hydrodynamic approach described above.

Lehmann-type effects exist in all phases that have a pseudoscalar chirality parameter $q_{0}$. These include other chiral liquid crystal phases (Brand and Pleiner 1988), like smectic $\mathrm{C}^{*}$ phases, where the in-plane director $c_{i}$ plays the role of $n_{i}$, but also phases with structural, ambidextrous chirality, like bent-core smectic $\mathrm{C}_{B 2}$ and tetrahedral $\mathrm{D}_{2}$ phases. Phases with ambidextrous helicity, like the tetrahedral $\mathrm{D}_{2 d}$ phase, however, are achiral and do not show classical Lehmann-type effects (Pleiner and Brand 2016).

The chiral cross-coupling terms in the dissipation function (17) not only lead to the Lehmann contributions in $Y_{i}^{D}$, but 
also to chiral additions to the dissipative entropy current, concentration current, and electric current, Eqs. 90-92,

$$
\begin{aligned}
j_{i}^{\sigma D} & =-\frac{\partial R}{\partial \nabla_{i} T}=q_{0} \psi_{\sigma} \epsilon_{i j k} n_{j} h_{k} \\
j_{i}^{c D} & =-\frac{\partial R}{\partial \nabla_{i} \mu_{c}}=q_{0} \psi_{c} \epsilon_{i j k} n_{j} h_{k} \\
j_{i}^{e D} & =\frac{\partial R}{\partial E_{i}}=-q_{0} \psi_{e} \epsilon_{i j k} n_{j} h_{k}
\end{aligned}
$$

Here $h_{i}=h_{i}^{n}+h_{i}^{c h}$, where $h_{i}^{n}$ is the nematic part and the chiral part $h_{i}^{c h}$ is given by Eq. 21 . The latter merely gives rise to chiral corrections $\sim q_{0}^{2}$ to heat conduction, diffusion, and electric conduction (and all the appropriate nematic cross effects in Eqs. 90-92).

On the other hand, rotating the director, e.g., by rotating electric (or magnetic) external fields (Cladis et al. 1995), a nonzero nematic molecular field $h_{i}^{n}=-\epsilon_{a} n_{j} E_{j} E_{k} \delta_{i k}^{\perp}$ exists due to the dielectric (diamagnetic) anisotropy energy. By Eqs. 24-26, this leads to finite contributions to the heat, concentration, and electric currents along $\boldsymbol{n} \times \boldsymbol{E}$, i.e., perpendicular to the rotation plane. This is the inverse Lehmann effect (Svenšek et al. 2008) that can be used as a microscopic pump for heat, particles, or charges. An experimental realization of these effects resides on a phase lag between the synchronous rotations of $\boldsymbol{E}$ and $\boldsymbol{n}$, which emerges due to dissipation resulting in a nonzero $\boldsymbol{h}^{n}$. Alternatively, a nonzero $\boldsymbol{h}^{n}$ can be also due to spatial inhomogeneities, e.g., created by flow as in the rotating needle experiment (Cladis et al. 1985). Recently an inverse Lehmann effect has been detected experimentally (Sato et al. 2017) for a rotating cholesteric droplet that produced a heat current (unidirectional heat transport). So far, all published data are compatible with the analysis of Svenšek et al. (2008) on using inverse Lehmann effects as a microscopic pump.

Clearly, it would be most desirable to have more experiments on the inverse Lehmann effect, which could then also be used-in combination with the results of measurements of the direct Lehmann effect-to determine the "static" $\left(\sim \tau_{c, \sigma, \rho}\right)$ and the "dissipative" chiral contributions $\left(\sim \psi_{c, \sigma, e}\right)$ separately.

We will now comment on some earlier descriptions of Lehmann effects. Leslie's description of the Lehmann effect (Leslie 1968) in cholesteric liquid crystals is compatible to ours. Since it does not consider time-reversal symmetry, it does not distinguish between reversible and irreversible effects. Nevertheless, Leslie's Lehmann contributions are obviously dissipative, as are ours. In Leslie's eq. (5.4), the heat current $q_{i}$ (=Tj $j_{i}^{\sigma}$ in our notation) and the molecular field $g_{i}$ ( $h_{i}$ in our notation) are given as linear functions of $\nabla_{i} T$ and of $N_{i}\left(=-Y_{i}\right.$ in our notation, cf. Eq. 72 and Leslie's eq. (2.1)). But a simple rearrangement brings them into the standard form, Eqs. 9 and 10. Since $g_{i}$ and $\nabla_{i} T$ are even under time reversal, Leslie's eq. (5.4) requires that his $q_{i}$ and $N_{i}$ also have to be even under time reversal with the result that only the dissipative parts of the currents are involved. Then, this fits to our description in Eqs. 18 and 24 and requires in Leslie's eq. (5.6) all inequalities $\leq$ and $\geq$ to be replaced by $<$ and $>$, respectively.

Nevertheless, there are some shortcomings. First, Leslie's description only includes thermal gradients as driving forces. Second, it considers only the dynamic chiral contributions omitting the static ones, Eq. 21. Since it does not employ linear irreversible thermodynamics, it uses two independent dissipative transport parameters for the direct and the inverse Lehmann effect ( $\lambda_{3}$ and $\kappa_{3}$ in Leslie's notation), while the existence of the dissipation function requires $T \lambda_{3}=-\kappa_{3}$. Moreover, the inverse effect is apparently not explicitly identified. With these caveats the two last lines of Leslie's eq. (5.4) are equivalent to our Eqs. 18 and 24.

The situation is somewhat different with the de Gennes description (de Gennes 1975). First, it includes not only temperature gradients, but also concentration gradients and electric fields. Second, de Gennes introduces time-reversal behavior to discuss this effect, but unfortunately in an unsatisfactory way. The statement (after his eq. (6.99)) that the electric current $\boldsymbol{j}^{e}$ ( $\boldsymbol{J}$ in his notation) is odd is true only for its reversible part. The electric charge density $\rho_{e}$ is even under time reversal and therefore $\partial \rho_{e} / \partial t$ has to be odd. As a consequence, the reversible part of the electric current $j_{i}^{e} R$ is odd as well, leaving the dynamic equation (74) invariant, while the dissipative part $j_{i}^{e D}$ is even and destroys the timereversal invariance of Eq. 74. Furthermore, he states, just before his eq. (5.27), that $\boldsymbol{N}$ and his $\boldsymbol{h}$ are odd (in the following, we call the latter $\boldsymbol{h}^{d G}$ in order to avoid confusion with our $\boldsymbol{h}$ ). However, the molecular field $h_{i}^{d G}$, which is $-h_{i}$ in our notation, [cf. Eq. 67 and his eq. (3.21)], has the same time-reversal behavior as the director $n_{i}$, which is even. In addition, $N_{i}$, which is $-Y_{i}$ in our notation, [cf. Eq. 72 and his eq. (5.22)], behaves odd only for the reversible part $Y_{i}^{R}$, but is even for the dissipative one, $Y_{i}^{D}$, similar to the case of $j_{i}^{e}$ discussed above.

Now, de Gennes describes the Lehmann effects in his eqs. (6.98) and (6.99). Omitting flow they read in his notation

$$
\begin{aligned}
h_{i}^{d G} & =h_{i}^{H}+v \epsilon_{i j k} n_{j} E_{k} \\
J_{i} & =\sigma_{\perp}^{E} E_{i}+\left(\sigma_{\|}^{E}-\sigma_{\perp}^{E}\right) n_{i} n_{j} E_{J}+v \epsilon_{i j k} n_{j} N_{k}
\end{aligned}
$$

where $h_{i}^{H}$ denotes the achiral (nematic) contribution. This is a rather unorthodox way of presenting the flux-force relation in linear irreversible thermodynamics, since Eq. 27 relates two forces with each other and Eq. 28 two fluxes. Nevertheless, he gets the Lehmann effect (almost) right. In his discussion of the Lehmann effect in subsection (6.3.4.2) 
he only uses Eq. 27 . With $N_{i}=\left(1 / \gamma_{1}\right) h_{i}^{d G}$, this leads to an expression which is equivalent to the second line of our Eq. 18, if $v=-q_{0} \gamma_{1} \psi_{e}$ is chosen. His rotation frequency eq. (6.104) then coincides with our expression in the last line of Eq. 23. Obviously, the Lehmann effect is related to the dissipative dynamics $Y_{i}^{D}$ and is not a reversible feature: Despite time running forward or backward, the amount of dissipated energy, the external driving field has to provide is the same. In de Gennes' description there are no "static" parts of the Lehmann effects, since he does not consider the chiral static contributions given in Eq. 21.

In Eq. 28, the electric conductivities ( $\sigma_{\perp}^{E}$ and $\sigma_{\|}^{E}$ ) clearly denote dissipative processes, and indeed, using the correct time-reversal behavior, $J_{i}$ and $N_{k}$ should be replaced by their dissipative parts (both reversible parts are then found to be zero). Replacing $N_{k}^{D}=-Y_{k}^{D}$ by $-\left(1 / \gamma_{1}\right) h_{k}$ via Eq. 18 , one can compare with our Eq. 26 leading to $v=q_{0} \gamma_{1} \psi_{e}$, which differs in sign from what we have got above from Eq. 27. The reason is that the signs for the $v$ terms have been chosen by de Gennes such that they cancel each other in the entropy production (which would be the right thing to do for reversible processes). Actually, for dissipative processes they should add up and Eq. 28 should read

$J_{i}^{D}=\sigma_{i j}^{E} E_{j}-v \epsilon_{i j k} n_{j} N_{k}^{D}$

This equation, not needed for the direct Lehmann effect, could lead to the inverse Lehmann effect, which was, however, not considered by de Gennes. Only when one measures both, the direct and the inverse Lehmann effect, one can detect the difference between Eqs. 28 and 29. Although Eqs. 27 and 29 can describe the ("dynamic" parts of the) Lehmann effects, the underlying concept is not suitable for a general description taking into account other hydrodynamic effects or variables, due to its special form relating forces with forces and fluxes with fluxes.

\section{Reversible dynamic coupling of flow to the nematic director dynamics}

As another example for the importance of time-reversal symmetry, we briefly discuss the reversible dynamic coupling between flow and the orientational degree of freedom, first, in nematics and, second, in pretransitional orientational fluctuations above the isotropic to nematic phase transition.

This reversible coupling is of the general form (15). Using the hydrodynamic description of nematic liquid crystals, it is given by Eqs. 82 and 83 describing flow alignment and backflow effects. In the continuum mechanics treatment of Leslie (1968II), this coupling is taken on equal footing with the (dissipative) viscosities as is seen in eqs. (5.31) and (5.32) of de Gennes (1975)

$$
\begin{aligned}
\sigma_{i j}^{d G}= & \alpha_{1} n_{i} n_{j} n_{k} n_{l} A_{k l}+\alpha_{4} A_{i j}+\alpha_{5} n_{i} n_{k} A_{j k} \\
& +\alpha_{6} n_{j} n_{k} A_{i k}+\alpha_{2} n_{i} N_{j}+\alpha_{3} n_{j} N_{i} \\
h_{i}^{d G}= & \gamma_{1} N_{i}+\gamma_{2} n_{j} A_{i j}
\end{aligned}
$$

with $\alpha_{2}+\alpha_{3}=\gamma_{2}$ and where $\sigma_{i j}^{d G}=-\sigma_{i j}, h_{i}^{d G}=-h_{i}$, and $N_{i}=-Y_{i}$ in our notation. In a symmetrized version of the stress tensor, only the sums $\alpha_{2}+\alpha_{3}$ and $\alpha_{5}+\alpha_{6}$ show up.

Since $h_{i}$ is even and $A_{i j}$ is odd under time reversal, Eq. 31 is split into a reversible part, $N_{i}^{R}=-\left(\gamma_{2} / \gamma_{1}\right) n_{j} A_{i j}$ and a dissipative one $N_{i}^{R}=\left(1 / \gamma_{1}\right) h_{I}^{d G}$. This is compatible with our description, Eqs. 83 and 93, for $\gamma_{2}=-\lambda \gamma_{1}$, a relation already given in eq. (5.40) of de Gennes (1975). Clearly, $\gamma_{2}$ (and therefore $\alpha_{2}+\alpha_{3}$ ) are reversible reactive parameters as is $\lambda$, and only $\alpha_{1}, \alpha_{4}$ and $\alpha_{5}+\alpha_{6}$ are dissipative parameters. Indeed, when de Gennes rewrites his equations in the hydrodynamic form, he finds that only three viscosities $v_{1,2,3}$ and $\gamma_{1}$ enter the dissipation function, cf. his eq. (5.41), while $\gamma_{2}$ does not, as is required for a reversible parameter. Thermodynamics then requires the dissipative transport parameters, $\nu_{1,2,3}$ and $\gamma_{1}$, all to be positive.

In terms of the $\alpha$ parameters used by de Gennes in his eq. (5.31) one gets, cf. his eqs. (5.39) and (5.34),

$2 v_{1}=\alpha_{1}+\alpha_{4}+\alpha_{5}+\alpha_{6}$

$2 v_{2}=\alpha_{4}$

$2 v_{3}=\alpha_{4}+\frac{1}{2}\left(\alpha_{5}+\alpha_{6}\right)-\frac{1}{2 \gamma_{1}} \gamma_{2}^{2}$

and the positivity requirements for $v_{1}$ and $v_{2}$ translate into appropriate positivity conditions on the dissipative $\alpha$ parameters. However, the positivity requirement $v_{3}>0$ would bound the value of the reversible reactive parameter $\gamma_{2}$ from above by the values of dissipative ones, or vice versa, it would bound dissipative parameters $\gamma_{1}\left[\alpha_{4}+\right.$ $\left.(1 / 2)\left(\alpha_{5}+\alpha_{6}\right)\right]$ by the value of a reactive one. Such a mixture of reversible reactive with irreversible transport parameters is physically unsound, since the microscopic origins of reversible and irreversible parameters are completely different as is shown by the Mori-Zwanzig formalism recalled in Appendix B. Dissipation originates exclusively from the non-instantaneous response covered by the memory matrix, while reversible dynamics is related to instantaneous response from the frequency matrix and, in the case of the flow alignment parameter, also from the memory matrix. A general relation mixing reversible and irreversible parameters is not possible, since it would relate dissipative processes with the instantaneous dynamics, which is neither possible in the quantum mechanical nor in the classical particle dynamics. The conclusion is that the continuum mechanics approach does not correctly describe the positivity of dissipative processes in this case. 
Flow alignment effects due to the reversible coupling between flow and director rotations exist in all liquid crystal phases that possess at least one nematic degree of freedom, but also for complex fluids with a preferred direction that is described by a polar vector (Brand et al. 2006), a velocity (Pleiner et al. 2013), a magnetization (Jarkova et al. 2003), or a vorticity (Brand et al. 2011).

There is a related problem in the dynamics of the isotropic to uniaxial nematic phase transition with respect to the dynamic coupling of the nematic order parameter $Q_{i j}$ to flow, introduced by de Gennes (1971). We again show that it is crucial to correctly identify the time-reversal symmetry of the currents to get the correct thermodynamic restrictions on the dynamic material parameters.

Using the hydrodynamic method, we start with the Gibbs relation, Eq. 1,

$T d \sigma=d \varepsilon-v_{i} d g_{i}-\Phi_{i j} d Q_{i j}$

where $\Phi_{i j}$ is the thermodynamic conjugate quantity to $Q_{i j}$. Both are even under time reversal and under spatial inversion. Here, the energy $\varepsilon$ is the Landau free energy of the isotropic to nematic phase transition, a power series in $Q_{i j}$.

In the isotropic phase, the linearized dynamic equations, defining the fluxes, can be written as

$$
\begin{gathered}
\dot{g}_{i}=\nabla_{j} \tilde{\sigma}_{j i} \\
\dot{Q}_{i j}=R_{i j}
\end{gathered}
$$

which is written in a way that facilitates the comparison with de Gennes (1975). With the dynamic entropy equation (4) the entropy production can be written

$2 R=\Phi_{i j} R_{i j}+A_{i j} \tilde{\sigma}_{i j}$

which is identical to eq. (5.102) of de Gennes (1975). The second law of thermodynamic leads to the conditions

$$
\begin{aligned}
& 2 R= \Phi_{i j} R_{i j}^{D}+A_{i j} \tilde{\sigma}_{i j}^{D}>0 \\
& \Phi_{i j} R_{i j}^{R}+A_{i j} \tilde{\sigma}_{i j}^{R}=0
\end{aligned}
$$

for, respectively, the irreversible and reversible parts of the currents and quasi-currents. Since $\dot{g}_{i}\left(\dot{Q}_{i j}\right)$ is even (odd) under time reversal, $\tilde{\sigma}_{i j}^{R}$ and $R_{i j}^{D}$ are even and $\tilde{\sigma}_{i j}^{D}$ and $R_{i j}^{R}$ are odd under time reversal. This allows for the following expressions

$$
\begin{aligned}
R_{i j}^{R} & =-\frac{2 \mu}{v} A_{i j} & \text { and } & R_{i j}^{D} & =\frac{1}{v} \Phi_{i j} \\
\tilde{\sigma}_{i j}^{R} & =\frac{2 \mu}{v} \Phi_{i j} & \text { and } & \tilde{\sigma}_{i j}^{D} & =2 \tilde{\eta} A_{i j}
\end{aligned}
$$

The condition for the dissipative currents, Eq. 39, requires for the dissipative transport parameters $v>0$ and $\tilde{\eta}>0$, while Eq. 40 is fulfilled automatically and does not pose any restriction on the reactive reversible parameter $\mu$.
In de Gennes (1975), eqs. (5.104) and (5.105), the current - force relations are written as

$$
\begin{aligned}
\Phi_{i j} & =2 \mu A_{i j}+v R_{i j} \\
\tilde{\sigma}_{i j} & =2 \eta A_{i j}+2 \mu R_{i j}
\end{aligned}
$$

where all three material parameters are classified as viscosities. Equations 43 and 44 are compatible with Eqs. 41 and 42 for $\eta=\tilde{\eta}+2 \mu^{2} / \nu$. This comparison shows that $\mu$ is a reactive parameter and not a viscosity, and $\eta$ is an oxymoron with dissipative $(\tilde{\eta})$ and reversible parts $(\mu)$.

Now, de Gennes puts the full expressions for $\Phi_{i j}$ and $\tilde{\sigma}_{i j}$ into the dissipation function $R$, Eq. 38, requiring $R>0$. By this procedure, he gets in his eq. (5.106) the positivity conditions $v>0, v \eta>2 \mu^{2}$, and $\eta>0$. These restrictions are difficult to interpret physically, since the oxymoron quantity $\eta$ is involved. In terms of the physically relevant parameters, the three conditions are translated into $v>0$, $\tilde{\eta}>0$, and $v \tilde{\eta}>2 \mu^{2}$, respectively. Compared to the hydrodynamic treatment, the third condition is an additional restriction in de Gennes' description. As in the case of $v_{3}>0$, Eq. 34, this condition would bound the value of the reversible reactive parameter $\mu$ from above by the values of dissipative ones, or vice versa, it would bound dissipative parameters $v \tilde{\eta}$ by the value of a reactive one. As discussed above, based on the Mori-Zwanzig formalism (Appendix B), such a mixture of reversible reactive with irreversible transport parameters is physically unsound. Again, the continuum mechanics approach does not correctly describe the positivity of dissipative processes in this case.

Similar couplings between flow and orientational order can be found in macroscopic equations describing the dynamics close to phase transitions have been derived on the basis of linear irreversible thermodynamics for the isotropic-superfluid phase transition in ${ }^{3} \mathrm{He}$ (Liu 1975), the uniaxial-biaxial phase transition in nematic liquid crystals (Jacobsen and Swift 1982) and also the isotropic-uniaxial nematic phase transition incorporating the effects of electric fields and spatial inhomogeneities Brand (1986, 1986II).

\section{Dynamic thermo-mechanical effects in textured nematic liquid crystals}

Hydrodynamics has been used to point out the existence of reversible cross-couplings between flow and temperature gradients (Brand and Pleiner 1987). They require the existence of director textures and explicitly take the form

$$
\begin{aligned}
\sigma_{i j}^{R} & =\cdots+\alpha_{i j k l p}^{(\sigma)}\left(\nabla_{k} T\right)\left(\nabla_{l} n_{p}\right) \\
j_{k}^{\sigma R} & =-\alpha_{i j k l p}^{(\sigma)}\left(\nabla_{i} v_{j}\right)\left(\nabla_{l} n_{p}\right)
\end{aligned}
$$

where the ... mean the expression given in Eq. 82. In addition, there are similar electro- and diffuso-mechanical 
effects replacing the temperature gradient by an electric field or a concentration gradient, and the heat current by the electric current or the concentration current, respectively (Brand and Pleiner 1987).

The thermo-mechanical coupling contributions are reversible, since the right-hand side of Eq. 45 is even under time reversal, as is $\dot{g}_{i}$, while the right-hand side of Eq. 46 is odd, as is $\dot{\sigma}$, cf. Eqs. 70 and 71 and the discussion after (80). Therefore, they must give rise to zero entropy production $R \equiv 0$, i.e., they must fulfill (81). Indeed, the opposite sign in Eq. 45 compared to Eq. 46 just guarantees this.

We note that the structure of the terms implies that the $\alpha$ tensors must contain an odd number of $\boldsymbol{n}$. Under this condition each $\alpha_{i j k l p}$ tensor contains ten independent coefficients

$$
\begin{aligned}
\alpha_{i j k l p}= & \alpha_{1}\left(n_{i} \delta_{j k}^{\perp} \delta_{l p}^{\perp}+n_{j} \delta_{i k}^{\perp} \delta_{l p}^{\perp}\right)+\alpha_{2} n_{k} \delta_{i j}^{\perp} \delta_{l p}^{\perp} \\
& +\alpha_{3}\left(n_{i} \delta_{j l}^{\perp} \delta_{k p}^{\perp}+n_{j} \delta_{i l}^{\perp} \delta_{k p}^{\perp}\right)+\alpha_{4} n_{l} \delta_{i j}^{\perp} \delta_{k p}^{\perp} \\
& +\alpha_{5}\left(n_{i} \delta_{j p}^{\perp} \delta_{k l}^{\perp}+n_{j} \delta_{i p}^{\perp} \delta_{k l}^{\perp}\right)+\alpha_{6} n_{i} n_{j} n_{l} \delta_{k p}^{\perp} \\
& +\alpha_{7}\left(n_{k} \delta_{i l}^{\perp} \delta_{p j}^{\perp}+n_{k} \delta_{i p}^{\perp} \delta_{j l}^{\perp}\right)+\alpha_{8} n_{i} n_{j} n_{k} \delta_{l p}^{\perp} \\
& +\alpha_{9} n_{l}\left(\delta_{i k}^{\perp} \delta_{p j}^{\perp}+\delta_{i p}^{\perp} \delta_{j k}^{\perp}\right) \\
& +\alpha_{10} n_{k} n_{l}\left(\delta_{i p}^{\perp} n_{j}+\delta_{j p}^{\perp} n_{i}\right)
\end{aligned}
$$

Already in Akopyan and Zeldovich (1984) thermomechanical effects in deformed nematics have been suggested. Without invoking time reversal, they erroneously assumed that those effects are dissipative and can be derived from a dissipation function, cf. their eq. A4. Such a "dissipation" function would have the form $R^{\text {thm }}=$ $\alpha_{i j k l p} A_{i j}\left(\nabla_{k} T\right)\left(\nabla_{l} n_{p}\right)$ and is given in the Appendix of Hakobyan et al. (2004).

If one uses $R^{t h m}$ as a dissipation function and derives the currents by partial differentiation (as would be the correct procedure, if the currents were dissipative, Eqs. 89 and 90), one would get the same heat current as Eq. 46, but in the stress tensor the $\alpha$ term would have the same sign as in the heat current, in contrast to our description, Eq. 45. This means the two approaches, Brand and Pleiner (1987) and Akopyan and Zeldovich (1984) are not equal, but the difference becomes manifest only, if both, the direct thermomechanical effect in the heat current and the inverse effect in the stress tensor are considered. The claim of Hakobyan et al. (2004) that the two descriptions are equal, is wrong, since they overlooked that our definition of the stress tensor differs from theirs by a sign (in our case $\sigma_{i j}^{D}=-\partial R / \partial A_{i j}$ while they use $\left.\sigma_{i j}^{R}=\partial R^{t h m} / \partial A_{i j}\right)$.

However, the discrepancy is much deeper. Since $A_{i j}$ (or the velocity $v_{i}$ ) is odd under time reversal, the function $R^{t h m}$ is odd, and can never be used as a dissipation function. If $R^{t h m}$ is positive for a given situation, it becomes negative, when the velocities are reversed. Such a behavior would grossly violate the second law of thermodynamics that requires the entropy production to be non-negative. Indeed, since the thermo-mechanical effects are reversible, their entropy production is zero, and from a function that is zero you can never derive currents by partial differentiation.

\section{Thermodynamic forces and currents in ferronematics and ferromagnetic nematics}

Ferronematics are suspensions of ferromagnetic particles in a nematic liquid crystal without magnetic order in equilibrium. If there is a finite magnetization in equilibrium, they are called ferromagnetic nematics. We restrict ourselves to the uniaxial case, where the director and the magnetization are parallel in equilibrium. In the following, the complex interplay of nematic, magnetic, and flow dynamics will be discussed and we compare critically the continuum mechanics approach by Raikher and Stepanov $(1996,1999)$ with the hydrodynamic approach by Jarkova et al. (2001, 2003).

The magnetization $\boldsymbol{M}$ is the additional degree of freedom compared to ordinary nematic liquid crystals. The nematic Gibbs relation (65) has to be generalized appropriately (Jarkova et al. 2003)

$d \varepsilon=T d \sigma+\mu d \rho+v_{i} d g_{i}+H_{i} d B_{i}+h_{i}^{M} d M_{i}+h_{i}^{n} d n_{i}$

containing changes of the magnetization $d M_{i}$, with $h_{i}^{M}$, the magnetic molecular field, as the conjugate thermodynamic force. To accommodate external fields we have added $B_{i}$, the magnetic flux density with its conjugate $H_{i}$, the magnetic Maxwell field, and electrodynamics is neglected.

The hydrodynamic equations for the density of linear momentum $g_{i}$, the director $n_{i}$ and the magnetization $M_{i}$ read, cf. Eqs. 70 and 72

$$
\begin{array}{r}
\dot{g}_{i}+\nabla_{j}\left(v_{j} g_{i}+p \delta_{i j}+\sigma_{i j}^{t h}+\sigma_{i j}\right)=0 \\
\dot{n}_{i}+v_{j} \nabla_{j} n_{i}+(\boldsymbol{n} \times \boldsymbol{\omega})_{i}+Y_{i}=0 \\
\dot{M}_{i}+v_{j} \nabla_{j} M_{i}+(\boldsymbol{M} \times \boldsymbol{\omega})_{i}+X_{i}=0
\end{array}
$$

with $\quad \sigma_{i j}^{t h}=-B_{j} H_{i}-\frac{1}{2}\left(M_{j} h_{i}^{M}-M_{i} h_{j}^{M}\right)$

$$
-\frac{1}{2}\left(n_{j} h_{i}^{n}-n_{i} h_{j}^{n}\right)+\Phi_{k j} \nabla_{i} n_{k}
$$

where $\omega_{i}=\frac{1}{2} \epsilon_{i j k} \nabla_{j} v_{k}$ is the vorticity. The thermodynamic contribution to the stress tensor, $\sigma_{i j}^{t h}$, can be symmetrized by a standard procedure (Martin et al. 1972).

The parts of the currents shown explicitly in Eqs. 4952 are reversible and material-independent and are given by general symmetry and thermodynamic principles (Pleiner and Brand 1996), like transformation behavior under translations (convective terms) or rotations (co-rotational 
terms, $\sim \boldsymbol{\omega})$ and by the requirement of zero entropy production $(R=0)$.

The phenomenological currents in Eqs. 49-51, $\sigma_{i j}$, $Y_{i}$ and $X_{i}$ are split into dissipative (superscript $D$ ) and reversible (superscript $R$ ) parts, like in Eq. 5. They are obtained using the transformation behavior under spatial inversion and time reversal of the quantities involved. Since the magnetization $M_{i}$ and the magnetic field $h_{i}^{M}$ are odd under time reversal (in contrast to $n_{i}$ and $h_{i}^{n}$ ), but even under spatial inversion, the reversible currents have the form (Jarkova et al. 2003)

$\sigma_{i j}^{R}=-\frac{1}{2} \lambda_{k j i} h_{k}^{n}-c_{k i j}^{R}(M) h_{k}^{M}-v_{i j k l}^{R}(M) A_{k l}$

$Y_{i}^{R}=-\frac{1}{2} \lambda_{i j k} A_{j k}+\left(\gamma^{-1}\right)_{i j}^{R}(M) h_{j}^{n}+\chi^{R}\left(\boldsymbol{n} \times \boldsymbol{h}^{M}\right)_{i}$

$X_{i}^{R}=b_{i j}^{R}(M) h_{j}^{M}+\chi^{R}\left(\boldsymbol{n} \times \boldsymbol{h}^{n}\right)_{i}-c_{i j k}^{R}(M) A_{j k}$

with $A_{i j}=\frac{1}{2}\left(\nabla_{i} v_{j}+\nabla_{j} v_{i}\right)$ and the nematic flow alignment tensor $\lambda_{i j k}$, Eq. 85 containing the flow alignment parameter $\lambda$.

The reversible couplings between director and magnetization provided by $\chi^{R}$ lead to zero entropy production due to their tensorial structure, $h_{i}^{n}\left(\boldsymbol{n} \times \boldsymbol{h}^{M}\right)_{i}+h_{i}^{M}\left(\boldsymbol{n} \times \boldsymbol{h}^{n}\right)_{i}=0$, rather than due to different signs as in the case of Eq. 15.

The magnetization-dependent tensors $\left(\gamma^{-1}\right)_{i j}^{R}(M)$, $b_{i j}^{R}(M), v_{i j k l}^{R}(M)$, and $c_{i j k}^{R}(M)$ are all odd functions of the magnetization. Throughout the rest of this section, we will take into account only terms linear in the magnetization $M_{i}$. The second rank tensors, i.e. the reversible analogues of director relaxation $\left(\gamma^{-1}\right)_{i j}^{R}(M)$ in Eq. 54 and the magnetization relaxation $b_{i j}^{R}(M)$ in Eq. 55, are both of the form

$$
\begin{aligned}
\kappa_{i j}^{R}(M)= & \kappa_{1}^{R} \epsilon_{i j k} M_{k}+\kappa_{2}^{R} \epsilon_{i j k} n_{k} n_{p} M_{p} \\
& +\kappa_{3}^{R}\left(\epsilon_{i p q} M_{p} n_{q} n_{j}-\epsilon_{j p q} M_{p} n_{q} n_{i}\right)
\end{aligned}
$$

They are antisymmetric, $\kappa_{i j}^{R}(M)=-\kappa_{j i}^{R}(M)$, according to Onsager's relation $\kappa_{i j}^{R}(M)=\kappa_{j i}^{R}(-M)$ and give rise to zero entropy production (Jarkova et al. 2001).

The third-rank tensor $c_{i j k}^{R}$ in Eqs. 53 and 55, describing dynamic cross-couplings between flow and magnetization, is symmetric in the last two indices and reads

$$
\begin{aligned}
c_{i j k}^{R}(M)= & c_{1}^{R} M_{i} n_{j} n_{k}+c_{2}^{R}\left(\delta_{i j} M_{k}+\delta_{i k} M_{j}\right) \\
& +c_{3}^{R} M_{i} \delta_{j k}+c_{4}^{R} n_{i} M_{p} n_{p} \delta_{j k} \\
& +c_{5}^{R}\left(n_{i} M_{j} n_{k}+n_{i} M_{k} n_{j}\right) \\
& +c_{6}^{R} n_{i} M_{p} n_{p} n_{j} n_{k}
\end{aligned}
$$

The fourth-rank tensor $v_{i j k l}^{R}(M)$ of Eq. 53, describing a selfcoupling between different components of the flow field, is not needed here. Its explicit form is given by eq. B3 of Jarkova et al. (2003).

The dissipative parts of the currents read

$$
\begin{aligned}
\sigma_{i j}^{D} & =-v_{i j k l}^{D} A_{k l}-\lambda_{k i j}^{D}(M) h_{k}^{n}-c_{k i j}^{D} h_{k}^{M} \\
Y_{i}^{D} & =\frac{1}{\gamma_{1}} \delta_{i j}^{\perp} h_{j}^{n}+\chi_{i j}^{D}(M) h_{j}^{M}+\lambda_{i j k}^{D}(M) A_{j k} \\
X_{i}^{D} & =b_{i j}^{D} h_{j}^{M}+\chi_{j i}^{D}(M) h_{j}^{n}+c_{i j k}^{D} A_{j k}
\end{aligned}
$$

where $1 / \gamma_{1}$ is associated with director relaxation or diffusion, $b_{i j}^{D}$ is of the standard uniaxial form, $b_{i j}^{D}=b_{\perp}^{D} \delta_{i j}^{\perp}+$ $b_{\|}^{D} n_{i} n_{j}$, describing the longitudinal and transverse relaxation of the magnetization, while $c_{i j k}^{D}=c^{D}\left(\epsilon_{i m k} n_{m} n_{j}+\right.$ $\left.\epsilon_{i m j} n_{m} n_{k}\right)$ provides a dissipative coupling between flow and orientation of the magnetization.

The dissipative second rank tensor $\chi_{i j}^{D}$ in Eqs. 59 and 60, coupling the director and the magnetization dynamics, has a form different from that of the reversible ones (56), since it has to contain an odd number of $\boldsymbol{n}$ 's,

$\chi_{i j}^{D}(M)=\chi_{1}^{D} \delta_{i k}^{\perp} M_{k} n_{j}+\chi_{2}^{D} \delta_{i j}^{\perp} M_{k} n_{k}$

The third-rank tensor $\lambda_{i j k}^{D}$ in Eqs. 58 and 59, the dissipative analogue of the reversible flow alignment tensor, is symmetric w.r.t. the last two indices and transverse to $\boldsymbol{n}$ in the first index,

$$
\begin{aligned}
\lambda_{i j k}^{D}(M)= & \lambda_{1}^{D}\left(\delta_{i q}^{\perp} \epsilon_{p j q} M_{p} n_{k}+\delta_{i q}^{\perp} \epsilon_{p k q} M_{p} n_{j}\right) \\
& +\lambda_{2}^{D}\left(\delta_{i k}^{\perp} M_{p} \epsilon_{p j q} n_{q}+\delta_{i j}^{\perp} M_{p} \epsilon_{p k q} n_{q}\right) \\
& +\lambda_{3}^{D}\left(M_{j} \epsilon_{i p k} n_{p}+M_{k} \epsilon_{i p j} n_{p}\right) \\
& +\lambda_{4}^{D}\left(M_{q} n_{q} n_{j} \epsilon_{i p k} n_{p}+M_{q} n_{q} n_{k} \epsilon_{i p j} n_{p}\right) \\
& +\lambda_{5}^{D} M_{p} \epsilon_{p i q} n_{q} n_{j} n_{k}+\lambda_{6}^{D} M_{p} \epsilon_{p i q} n_{q} \delta_{j k}^{\perp}
\end{aligned}
$$

As a reference for the comparison following below, we list the couplings of Eqs. 53-62 specific for ferronematics:

1: Dynamic reversible cross-coupling between director and magnetization $\sim \chi^{R}$

2: Dynamic reversible cross-coupling between flow and magnetization $\sim c_{k i j}^{R}$

3: Dynamic reversible self-coupling of the director $\sim$ $\left(\gamma^{-1}\right)_{i j}^{R}$

4: Dynamic reversible self-coupling of the magnetization $\sim b_{i j}^{R}$

5: Dynamic dissipative cross-coupling between director and magnetization $\sim \chi_{i j}^{D}$

6: Dynamic dissipative cross-coupling between flow and magnetization $\sim c_{k i j}^{D}$

7: Dynamic dissipative cross-coupling between flow and director $\sim \lambda_{k i j}^{D}$ 
8: Dynamic dissipative self-coupling of the magnetization $\sim b_{i j}^{D}$

In the continuum mechanics approach of ferronematics by Raikher and Stepanov (1996, 1999), the dynamics of the nematic director field, Eq. 2 of Raikher and Stepanov (1996), is given in our notation by

$\epsilon_{i j k} n_{j}\left(N_{k}+\frac{\gamma_{2}}{\gamma_{1}} A_{k l} n_{l}\right)-\frac{1}{\gamma_{1}}\left(\boldsymbol{n} \times \boldsymbol{h}^{n}\right)_{i}=0$

where $\boldsymbol{N}=\dot{\boldsymbol{n}}-\boldsymbol{\omega} \times \mathbf{n}$. The rotational "viscosity" coefficients $\gamma_{1}$ and $\gamma_{2}$ are as in the continuum mechanics approach of uniaxial nematics and $\boldsymbol{h}^{n}$ is the molecular field of the director (de Gennes 1975).

For the dynamics of the magnetization, Raikher and Stepanov suggest an equation of a very similar structure as for the director

$\epsilon_{i j k} m_{j}\left(M_{k}^{R S}+\frac{\gamma_{2 \rho}}{\gamma_{1 \rho}} A_{k l} m_{l}\right)-\frac{1}{\gamma_{1 \rho}}\left(\boldsymbol{m} \times \boldsymbol{h}^{m}\right)_{i}=S_{i}$

where $\boldsymbol{M}^{R S}=\dot{\boldsymbol{m}}-\boldsymbol{\omega} \times \mathbf{m}$, with the normalized magnetization $\boldsymbol{m}=\boldsymbol{M} /|\boldsymbol{M}|$ and where $\boldsymbol{S}$ is a source term arising from a surface energy that does not contain any reversible or dissipative dynamic transport parameters.

Now, we are ready to compare the hydrodynamic approach with the continuum mechanics approach by Raikher and Stepanov. Equation 63 is the standard nematic director equation in the continuum mechanics description. It is shown in "Reversible dynamic coupling of flow to the nematic director dynamics" that the $\gamma_{1}$ is the same as in Eq. 59, while $\gamma_{2}$ is related to the flow alignment parameter $\lambda$ in Eq. 54 by $\gamma_{2}=-\lambda \gamma_{1}$. Since both $N_{k}$ and $A_{k l} n_{l}$ in Eq. 63 have the same time-reversal behavior (odd), it is obvious that $\gamma_{2}$ is a reversible reactive parameter.

In the magnetization dynamics, Eq. 64, $\gamma_{1 \rho}$ corresponds to the transverse magnetization relaxation $1 / b_{\perp}^{D}$ in Eq. 60 , while $\gamma_{2 \rho}$ is related to $c_{2}^{R}$ in Eq. 55 by $\gamma_{2 \rho}=-2 \gamma_{1 \rho} c_{2}^{R}$. Since both, $M_{k}^{R S}$ and $A_{k l} m_{l}$ in Eq. 64 are even under time reversal, $c_{2}^{R}$ is a reversible reactive parameter. All other parameters contained in the $c_{i j k}^{R}$ tensor of Eq. 57 do not show up in the continuum mechanics approach.

The two parameters $\gamma_{1 \rho}$ and $\gamma_{2}$, respectively, correspond to the points 8: and 2: in the list provided above. All other couplings listed there, points 1: and 3: to 7: are completely missing in the continuum mechanics approach. Those couplings owe their existence to the fact that $\boldsymbol{n}$ and $\boldsymbol{m}$ have opposite time-reversal behavior, demonstrating the importance of this symmetry when setting up hydrodynamic equations.

There is no expression for the stress tensor given by Raikher and Stepanov. However, the flow couplings $\sim \gamma_{2}$ and $\sim \gamma_{2} \rho$ require counter terms in the stress tensor in order to guarantee zero entropy production, cf. Eq. 53. Even if one disregards flow, the couplings number 3: to 5: are still missing. This clearly contradicts the recent claim by Boychuk et al. (2016), that the two approaches, namely, the one by Raikher and Stepanov on the one hand and by Jarkova et al. on the other would be equivalent without flow field.

We point out that the couplings missing in the continuum mechanics approach are important for the understanding of recent magneto-optic experiments. A couple of years ago uniaxial ferromagnetic nematics have been synthesised and their physical properties have been characterised (Mertelj et al. 2013, 2014; Sahoo et al. 2015). Very recently, it has become clear experimentally and theoretically (Potisk and et al. 2017, 2018) that dynamic cross-coupling terms between the director and the magnetization of the type discussed above (point 5:, $\sim \chi_{i j}^{D}$ ), are crucial to explain the experimental observations. It must be emphasized that for the considered materials these couplings are far from being just corrections - if they are missing, the dynamics takes a qualitatively different path, one that is not observed in the experiment. As has been shown by Potisk and et al (2017), it is exactly the dissipative cross coupling $\chi_{2}^{D}$ of Eq. 61 that is responsible for the observed, unusually fast magnetoelectric response. This result opens the door to applications of these new ferromagnetic liquids in devices requiring small electric and/or magnetic fields for controlling large magneto-optic effects.

\section{Summary}

Using the hydrodynamic description, we have shown that the behavior under time reversal plays an important role for the macroscopic description of soft matter systems. First, we discuss Lehmann-type effects in chiral liquid crystals that have a nematic degree of freedom. We show that the direct Lehmann effect, director rotations driven by external fields perpendicular to the director, generally has two origins. It arises due to a chiral dissipative dynamic cross-coupling (together with achiral static susceptibilities) as well as due a chiral static cross-coupling (together with an achiral dissipative dynamic parameter), which are sometimes called "dynamic" and "static" Lehmann effect, respectively. In the continuum mechanics approaches, the latter is missing and the Lehmann effect is erroneously related to the reversible dynamics. The latter point is crucial for the inverse Lehmann effect, where driven director rotations lead to temperature, concentration, or electric currents perpendicular to the director (heat, particle, or charge transportation across the rotation plane). This inverse Lehmann effect is governed by the same dissipative dynamic cross-coupling parameter that is responsible for 
the direct "dynamic" Lehmann effect. In the continuum mechanics approaches, the inverse Lehmann effect is generally overlooked, although it is present in their formulas. However, due to their assumption of reversibility, it comes with the wrong sign. This difference to the hydrodynamic description becomes manifest in recent experiments that are able to measure the direct and the inverse Lehmann effect for the same system.

Secondly, the flow alignment effect, the oblique orientation of a director in a steady shear flow is described in hydrodynamics by a reversible dynamic reactive parameter. In continuum mechanics approaches, the effect is assumed to be a dissipative one and, as a result, there is a positivity condition (common for irreversible transport parameters) that contains a combination of reversible and irreversible parameters. Such a restriction of a reversible parameter by dissipative ones (and vice versa) is impossible, since microscopically irreversible parameters are connected to the non-instantaneous response and a reversible one (at least partly) to the instantaneous response, and both cannot mix. In the hydrodynamic picture, the positivity conditions only apply to dissipative parameters, while the reversible one is not restricted. This applies also to nematic fluctuations above the isotropic to nematic phase transition and to systems with a preferred direction other than a director, e.g., a magnetization.

Thirdly, we discuss thermo-mechanical (and electromechanical) effects in textured nematic liquid crystals that are of interest for engineering applications. These are crosscouplings between flow and temperature gradients (and electric fields) that are reversible according to their timereversal behavior. In the literature, it is claimed that such effects are irreversible, an error that becomes manifest, if both, the direct thermo-mechanical effect in the heat current and the inverse effect in the stress tensor are measured.

Finally, we compare the hydrodynamic description of ferronematics (and ferromagnetic nematics) with an approach that uses Leslie-Ericksen-type equations for the nematic as well as for the magnetic degree of freedom. This continuum mechanics approach lacks many (reversible and irreversible) dynamic cross-couplings among the nematic and the magnetic aspects of those materials. These crosscouplings are possible due to the different time-reversal behavior of a director and a magnetization. They are important as has been shown recently in magneto-optic measurements (Potisk and et al 2017; 2018).

Funding Information Open access funding provided by Max Planck Society. We acknowledge partial support by the Deutsche Forschungsgemeinschaft through the Schwerpunktprogramm SPP 1681 'Feldgesteuerte Partikel-Matrix-Wechselwirkungen: Erzeugung, skalenübergreifende Modellierung und Anwendung magnetischer Hybridmaterialien'. In addition, D.S. acknowledges the support of the Slovenian Research Agency through Grants J1-7435, J1-7441.
Open Access This article is distributed under the terms of the Creative Commons Attribution 4.0 International License (http://creativecommons. org/licenses/by/4.0/), which permits unrestricted use, distribution, and reproduction in any medium, provided you give appropriate credit to the original author(s) and the source, provide a link to the Creative Commons license, and indicate if changes were made.

\section{Appendix A: Application to nematic liquid crystals}

For uniaxial nematic liquid crystals, a fluid system with spontaneously broken continuous rotational symmetry (Forster 1975), the hydrodynamic equations have been derived in the framework of linear irreversible thermodynamics (Forster et al. 1971). As additional hydrodynamic variables compared to a simple fluid one has the rotation of the director, $n_{i}$, with $n_{i}^{2}=1$ and the $n_{i} \rightarrow-n_{i}$ equivalence, since one cannot distinguish between head and tail in a nematic liquid crystal (quadrupolar orientational order). In addition, we consider a concentration field $c$ to allow for mixtures and lyotropic systems and an electric polarization via the electric displacement field $D_{i}$.

The Gibbs relation (1) for uniaxial nematics reads

$$
\begin{aligned}
T d \sigma= & d \varepsilon-\mu d \rho-\rho \mu_{c} d c-\boldsymbol{v} \cdot d \boldsymbol{g}-h_{i}^{\prime} d n_{i}-\phi_{i j} d \nabla_{j} n_{i} \\
& -E_{i} d D_{i}
\end{aligned}
$$

or equivalently

$T d \sigma=d \varepsilon-\mu d \rho-\rho \mu_{c} d c-\boldsymbol{v} \cdot d \boldsymbol{g}-h_{i} d n_{i}-E_{i} d D_{i}$

where $\varepsilon$ is the energy density, $\rho$ the (mass) density, $\sigma$ the entropy density, and $g_{i}$ the density of linear momentum. The thermodynamic forces (16) introduced by Eq. 66 are chemical potential $\mu$, osmotic pressure $\mu_{c}$, temperature $T$, velocity $v_{i}$, electric field $E_{i}$, and the molecular field of the director

$h_{i}=\frac{\delta \varepsilon}{\delta n_{i}}=\frac{\partial \varepsilon}{\partial n_{i}}-\nabla_{j} \frac{\partial \varepsilon}{\partial \nabla_{j} n_{i}}=h_{i}^{\prime}-\nabla_{j} \phi_{i j}$

The variables and conjugates that are even under time reversal are $T, \sigma, \varepsilon, \mu, \rho, c, \mu_{c}, D_{i}, E_{i}, h_{i}$ and $n_{i}$, while $v_{i}$ and $g_{i}$ are odd.

For the dynamic equations (2), we have (for details cf. Pleiner and Brand (1996))

$$
\begin{aligned}
& \dot{\rho}+\nabla_{i} g_{i}=0 \\
& \dot{c}+v_{i} \nabla_{i} c+\frac{1}{\rho} \nabla_{i} j_{i}^{c}=0 \\
& \dot{g}_{i}+\nabla_{j}\left(\delta_{i j} p+v_{i} g_{j}+\sigma_{i j}^{t h}+\sigma_{i j}\right)=0 \\
& \dot{\sigma}+\nabla_{i}\left(v_{i} \sigma\right)+\nabla_{i} j_{i}^{\sigma}=\frac{2 R}{T} \\
& \dot{n}_{i}+v_{j} \nabla_{j} n_{i}+(\boldsymbol{n} \times \boldsymbol{\omega})_{i}+Y_{i}=0 \\
& \dot{\varepsilon}+\nabla_{i} v_{i}(\varepsilon+p)+\nabla_{i} j_{i}^{\varepsilon}=0 \\
& \dot{\rho}_{e}+\nabla_{i}\left(v_{i} \rho_{e}\right)+\nabla_{i} j_{i}^{e}=0
\end{aligned}
$$


with $\rho_{e}$ and $j_{i}^{e}$ the charge density and the electric current, respectively, $p=-\varepsilon+\mu \rho+\rho \mu_{c} c+\boldsymbol{v} \cdot \boldsymbol{g}$ the pressure, $\omega_{i}=(1 / 2) \epsilon_{i j k} \nabla_{j} v_{k}$ the vorticity, and where we have made explicit the convective terms associated with the hydrodynamic variables. The thermodynamic contribution to the stress tensor, $\sigma_{i j}^{t h}=\phi_{k j} \nabla_{i} n_{k}+(1 / 2)\left(n_{i} h_{j}-n_{j} h_{i}\right)$ can be symmetrized by a standard procedure (Martin et al. 1972). In $\sigma_{i j}^{t h}$ and in the pressure, there are some electric field effects (Pleiner and Brand 1996), which are irrelevant for the present purpose. The dissipation function $R=0$ for reversible processes and $R>0$ for irreversible ones. There is no need to write down an explicit dynamic equation for the energy density, since it follows from the other dynamic equations via the Gibbs relation. The mass current in Eq. 68 does not have an irreversible part, since it is the momentum density $g_{i}=\rho v_{i}$.

Splitting up the total currents into reversible $(\mathrm{R})$ and irreversible (D) parts like in Eq. 5

$$
\begin{aligned}
\sigma_{i j} & =\sigma_{i j}^{R}+\sigma_{i j}^{D} \\
\boldsymbol{j}^{\sigma} & =\boldsymbol{j}^{\sigma R}+\boldsymbol{j}^{\sigma D} \\
\boldsymbol{j}^{c} & =\boldsymbol{j}^{c R}+\boldsymbol{j}^{c D} \\
\boldsymbol{j}^{e} & =\boldsymbol{j}^{e R}+\boldsymbol{j}^{e D} \\
\boldsymbol{j}^{\varepsilon} & =\boldsymbol{j}^{\varepsilon R}+\boldsymbol{j}^{\varepsilon D} \\
Y_{i} & =Y_{i}^{R}+Y_{i}^{D}
\end{aligned}
$$

we get a definite time-reversal behavior for the currents, since the reversible ones behave the same way as the time derivative of the appropriate variable, while the dissipative ones have the opposite time-reversal behavior. In particular $\sigma_{i j}^{R}, j_{i}^{\sigma D}, j_{i}^{c D}, j_{i}^{e D}$ and $Y_{i}^{D}$ are even, while $\sigma_{i j}^{D}, j_{i}^{\sigma R}, j_{i}^{c R}$, $j_{i}^{e R}$ and $Y_{i}^{R}$ are odd.

First, we summarise the reversible part of the dynamics following closely Forster et al. (1971) and Pleiner and Brand (1996). Inserting all dynamic equations, that is Eqs. 68-74 into Eq. 66 leads to the conditions, which the reversible parts of the currents and quasi-currents must satisfy to conserve entropy. They are, requiring $R \equiv 0$

$$
\begin{aligned}
& \nabla_{k}\left(j_{k}^{\varepsilon R}-v_{i} \sigma_{i k}^{R}-\Phi_{i k} Y_{i}^{R}-T j_{i}^{\sigma R}-j_{i}^{c R} \mu_{c}\right) \\
= & -j_{i}^{\sigma R} \nabla_{i} T-j_{i}^{c R} \nabla_{i} \mu_{c}-\sigma_{i j}^{R} \nabla_{j} v_{i} \\
& +h_{i} \delta_{i j}^{\perp} Y_{j}^{R}+j_{i}^{e R} E_{i}
\end{aligned}
$$

Defining the energy current density $j_{i}^{\varepsilon R}$ such that the total divergence of the first line vanishes, the remaining condition has the form of Eq. 7. Since there is no potential from which reversible currents can be obtained, one has to look for the most general expressions allowed by time reversal symmetry according to Eq. 6 and by spatial symmetry

$$
\begin{aligned}
\sigma_{i j}^{R} & =-\frac{1}{2} \lambda_{k j i} h_{k} \\
Y_{i}^{R} & =-\frac{1}{2} \lambda_{i j k} \nabla_{j} v_{k} \\
j_{i}^{\sigma R} & =0=j_{i}^{c R}=j_{i}^{e R}
\end{aligned}
$$$$
\text { with } \quad \lambda_{i j k}=\lambda\left(\delta_{i j}^{\perp} n_{k}+\delta_{i k}^{\perp} n_{j}\right)
$$

containing the flow alignment parameter $\lambda$, a reversible reactive parameter, whose sign is not fixed and whose value is not bounded. The reversible cross-coupling in Eqs. 82 and 83 comes with the same (symmetric) sign, since here we deal with the cross-coupling between a conserved and a non-conserved variable, contrary to the antisymmetric case of Eq. 15. In addition, the sequence of the indices of the coupling tensor in the two equations is different indicating the connection of thermodynamic conditions and spatial structures in anisotropic systems.

In the presence of director textures there are, additionally, reversible cross-couplings between flow and temperature gradient, concentration gradients and electric fields, which are discussed in "Dynamic thermo-mechanical effects in textured nematic liquid crystals" section.

For the dissipative nematic currents we proceed along the same lines as above for the reversible currents. The general positivity condition (8) according to the second law of thermodynamics leads for the dissipation function to

$$
\begin{aligned}
0<2 R_{n}= & -j_{i}^{\sigma D} \nabla_{i} T-j_{i}^{c D} \nabla_{i} \mu_{c}+j_{i}^{e D} E_{i} \\
& -\sigma_{i j}^{D} \nabla_{j} v_{i}+h_{i} \delta_{i j}^{\perp} Y_{j}^{D}
\end{aligned}
$$

and for the dissipative energy current we obtain

$j_{i}^{\varepsilon D}=T j_{i}^{\sigma D}+v_{j} \sigma_{j i}^{D}+\mu_{c} j_{i}^{c D}+\Phi_{i j} Y_{j}^{D}$

We also note that for the solution of boundary value problems for liquid crystals one uses specific boundary conditions such as planar, homeotropic or hybrid (planar on one bounding plate and homeotropic on the other) for the nematic director at top and bottom plate of a typical sample (compare, for example (de Gennes 1975; Rey 2010)).

To obtain the dissipative parts of the currents one can start with the dissipation function $R$ as a bilinear function of the forces. Taking into account the time-reversal symmetries of the forces (and of $R$ ) one gets

$$
\begin{aligned}
R_{n}= & \frac{1}{2 \gamma_{1}} h_{i} \delta_{i j}^{\perp} h_{j}+\frac{1}{2} v_{i j k l}\left(\nabla_{j} v_{i}\right)\left(\nabla_{l} v_{k}\right) \\
& +\frac{1}{2} \kappa_{i j}\left(\nabla_{i} T\right)\left(\nabla_{j} T\right)+\frac{1}{2} D_{i j}\left(\nabla_{i} \mu_{c}\right)\left(\nabla_{j} \mu_{c}\right) \\
& +D_{i j}^{T}\left(\nabla_{i} T\right)\left(\nabla_{j} \mu_{c}\right)+\frac{1}{2} \Sigma_{i j}^{E} E_{i} E_{j} \\
& +D_{i j}^{E} E_{i} \nabla_{j} \mu_{c}+\kappa_{i j}^{E} E_{i} \nabla_{i} T
\end{aligned}
$$


where the tensors associated with heat conductivity, $\kappa_{i j}$, diffusion $D_{i j}$, electric conductivity $\Sigma_{i j}^{E}$, thermoelectricity $\kappa_{i j}^{E}$, thermal diffusion $D_{i j}^{T}$, and the concentration-electric tensor $D_{i j}^{E}$ are of the usual uniaxial form, e.g. $\kappa_{i j}=$ $\kappa_{\perp} \delta_{i j}^{\perp}+\kappa_{\|} n_{i} n_{j}$, while the viscosity tensor $v_{i j k l}$ contains five independent viscous coefficients (Mason 1958), and there is only one rotational viscosity $\gamma_{1}$. Positivity of the entropy production leads to the requirement that $\gamma_{1}, \kappa_{\perp}, \kappa_{\|}, D_{\|}, D_{\perp}$, $\Sigma_{\|}^{E}$ and $\Sigma_{\perp}^{E}$ are all positive with corresponding additional requirements on the five viscous coefficients (compare, for example, the monograph of Forster (1975) or the Appendix of Pleiner and Brand (1996)).

The dissipative currents and quasi-currents are then obtained by taking functional derivatives of the dissipation function with respect to the forces

$$
\begin{aligned}
-\sigma_{i j}^{D} & =\frac{\partial R}{\partial \nabla_{j} v_{i}}=v_{i j k l} \nabla_{k} v_{l} \\
-j_{i}^{\sigma D} & =\frac{\partial R}{\partial \nabla_{i} T}=\kappa_{i j} \nabla_{j} T+D_{i j}^{T} \nabla_{j} \mu_{c}+\kappa_{i j}^{E} E_{i} \\
-j_{i}^{c D} & =\frac{\partial R}{\partial \nabla_{i} \mu_{c}}=D_{i j} \nabla_{j} \mu_{c}+D_{i j}^{T} \nabla_{j} T+D_{i j}^{E} E_{i} \\
j_{i}^{e D} & =\frac{\partial R}{\partial E_{i}}=\Sigma_{i j}^{E} E_{j}+D_{i j}^{E} \nabla_{j} \mu_{c}+\kappa_{i j}^{E} \nabla_{j} T \\
Y_{i}^{D} & =\frac{\partial R}{\partial h_{i}}=\frac{1}{\gamma_{1}} \delta_{i j}^{\perp} h_{j} .
\end{aligned}
$$

The hydrodynamic description of nematic liquid crystals is completed by providing an energy functional, $\varepsilon$, that leads to the static relations between the variables and the conjugates. In the nematic case, $\varepsilon$ is a sum of the isotropic liquid expression and the Frank gradient energy, including electric field effects. The former contains the scalar susceptibilities, specific heat, compressibility and thermal expansion etc., while the latter shows the three Frank constants $K_{1,2,3}$ describing splay, bend, and twist deformations of the director field and flexoelectricity. In addition, it contains the dielectric term and the effects of the concentration field $c$.

We close this review-type section by mentioning a qualitative difference in the viscous behavior between nematic and cholesteric liquid crystals. For nematics, one has the three so-called Miesowicz viscosities (Miesowicz 1935 , 1946) related to the three configurations under shear flow measured for a strong aligning magnetic or electric field for the director (de Gennes 1975): $\eta_{c}$ for the director parallel to the velocity gradient direction, $\eta_{b}$ for the director aligned in the velocity direction and $\eta_{a}$ for the director aligned in the vorticity direction. For usual rod-like nematics, one typically finds the inequalities (de Gennes 1975; Beens and de Jeu 1983) $\eta_{b}<\eta_{a}<\eta_{c}$. As has been elucidated in (Rey 2000), this situation changes qualitatively by considering cholesteric liquid crystals in the small frequency limit for aligned helical axis. Denoting the viscosity for the case of the helix oriented along the flow direction by $\eta_{f}$, for the helical axis oriented along the gradient direction of the velocity field by $\eta_{g}$ and for the helix along the vorticity direction by $\eta_{v}$, one obtains in the low frequency limit (Rey 2000) the inequalities $\eta_{g}<\eta_{v}<$ $\eta_{f}$.

\section{Appendix B: Instantaneous versus non-instantaneous contributions to reversible currents}

In the bulk part of this paper, we have focused on the phenomenological, symmetry-based analysis of reversible and irreversible currents. Here, we make use of a statistical mechanics approach pioneered by Forster (1974, 1974II, 1975) applying the Mori-Zwanzig formalism in the hydrodynamic regime. The Mori-Zwanzig projector formalism (Zwanzig 1960, 1961; Mori 1965) leads to dynamic equations for the correlation functions of the variables of choice by projecting out all irrelevant variables. By construction, this approach has automatically built-in linear irreversible thermodynamics (Forster 1975).

We summarise here very briefly the main results and refer for the details of the derivation as well as for the application to many different physical systems to the monograph of Forster (1975). The central object is the matrix of Kubo functions, $C_{k j}(z)$, defined as Laplace-transformed, equilibrium-averaged correlation functions

$C_{i j}(z)=\left\langle A_{i}\left|\frac{i}{z-L}\right| A_{j}\right\rangle$

where $L$ is the Liouville operator and $\left\langle A_{i} \mid B_{j}\right\rangle$ is the scalar product of the set of variables $A_{i}$ and $B_{j}$ in the proper Hilbert space. The main result is the complex dynamic matrix equation

$\left[z \delta_{i k}-\Omega_{i k}+i \Sigma_{i k}\right] C_{k j}(z)=i \beta^{-1} \chi_{i j}$

with $\beta^{-1}=k_{B} T$. The matrix of static correlation functions, $\chi_{i j}=\beta\left\langle A_{i} \mid A_{j}\right\rangle$ acts as the source term in Eq. 95. The matrix $\Omega_{i j} \equiv \omega_{i k} \chi_{k j}^{-1}$ is related to the frequency matrix $\omega_{i j}$ that describes the instantaneous response $\omega_{i j}=i \beta\left\langle\dot{A}_{i} \mid A_{j}\right\rangle$. Finally, $\Sigma_{i j}(z) \equiv \sigma_{i k}^{m}(z) \chi_{k j}^{-1}$ is related to the memory matrix $\sigma_{i j}^{m}(z)$ that describes the non-instantaneous response

$\sigma_{i j}^{m}(z)=\beta\left\langle\dot{A_{i}}\left|Q \frac{i}{z-Q L Q} Q\right| \dot{A_{j}}\right\rangle$

Here, the projection onto the relevant variables is manifest by $Q \equiv 1-P$, the complement of the projector operator

$P=\left|A_{i}\right\rangle \beta \chi_{i j}^{-1}\left\langle A_{j}\right|$

which is at the heart of this method. 
The main result (95) can be applied and compared to linear hydrodynamics (Forster 1975). The matrix of static susceptibilities $\chi_{i j}$ gives the static susceptibilities, e.g., the compressibility, specific heat, and thermal expansion, but also the superfluid density (for quantum fluids) and the Frank elastic constants (for nematic liquid crystals).

The frequency matrix $\omega_{i j}$ comprises the instantaneous response as the expectation value of an equal time commutator (quantum mechanically) or a Poisson bracket (classically) and contains only reversible effects, in particular, dynamic reactive parameters.

The memory matrix $\sigma_{i j}^{m}$ contains all dissipative transport parameters, like heat conductivity, shear and bulk viscosity, and (in liquid crystals) the nematic orientational viscosity. However, even in the hydrodynamic regime, the memory matrix can contain not only dissipative contributions, but also reversible ones. The first system for which this was found to be the case were uniaxial nematic liquid crystals (Forster 1974, 1974II, 1975), namely, for the flow alignment coefficient, $\lambda$, Eq. 85 . Therefore, $\lambda$ has two contributions, an instantaneous one from the real part of the frequency matrix $\omega_{i j}$, and a non-instantaneous one from the imaginary part of the memory matrix $\sigma_{i j}^{m}$. The antisymmetry of the latter part is sometimes called Casimir relation (Öttinger 2014). Nevertheless, $\lambda$ is a reversible, reactive parameter describing macroscopic reversible effects.

The situation is somewhat similar for the reversible coefficient $\chi^{R}$ describing the coupling between director and magnetization in Eqs. 54 and 55: it has a reversible contribution from the memory matrix, i.e., there is a Casimir contribution. In the case of $\chi^{R}$, both contributions $\left(\sim \omega_{i j}\right.$ and $\sim \sigma_{i j}^{m}$ ) do not vanish in the long wavelength limit.

Later this phenomenon was found also for superfluid ${ }^{3} \mathrm{He}-\mathrm{A}$ (Brand et al. 1979), superfluid ${ }^{3} \mathrm{He}-\mathrm{A}$ in high magnetic fields (Brand and Pleiner 1981II), superfluid ${ }^{3} P_{2}$ neutron star matter (Brand and Pleiner 1981III), superfluid ${ }^{3} \mathrm{He}-A_{1}$ (Brand and Pleiner 1981II), in superfluid ${ }^{3} \mathrm{He}-\mathrm{B}$ in high magnetic fields (Pleiner and Brand 1983), as well as in biaxial nematics (Brand and Pleiner 1981, 1982; Liu 1981) and in smectic C (Brand and Pleiner 1982III). It also arises for polar nematics (Brand et al. 2006) and in hexatic $\mathrm{F}$ and I phases (Pleiner 1984), as well as in the tetrahedral phases with preferred directions, D2d, D2, and S4 (Pleiner and Brand 2014, 2016).

The characteristic feature of all these hydrodynamic cases having reversible contributions from the memory matrix is that they either involve broken rotational symmetry in real space associated with angular momentum density as the generator of the broken rotational symmetry or, alternatively, the total angular momentum $\vec{J}=\vec{L}+\vec{S}$ with the spin $\vec{S}$ as it is the case for superfluid ${ }^{3} P_{2}$ neutron star matter (Brand H and Pleiner H 1981III).
The importance of this statistical mechanics approach in terms of correlation functions for linear hydrodynamics lies in the possibility to relate atomistic or coarse-grained numerical simulation data to the values of the hydrodynamic parameters. In the present context, however, we use this approach to argue in the "Reversible dynamic coupling of flow to the nematic director dynamics" that restrictive conditions such as inequalities between reversible and irreversible dynamic parameters cannot occur, due to their physically different origins.

For a long time, it is known (Forster et al. 1971; Forster 1974, 1974II, 1975) that the hydrodynamics of nematic liquid crystals has got a dynamic reversible "reactive" parameter, the flow alignment parameter, whose value depends on the material and is not fixed by invariance principles. The dynamics governed by this reversible reactive parameter does not generate entropy. For example, the homogeneous rotation of the director during the transient flow-aligning process does not contribute to dissipation, and neither does tumbling. Such reversible cross-coupling terms (between flow and the rotation of a preferred direction) leading to vanishing entropy production have also been found to arise for other systems, including smectic C (Martin et al. 1972), biaxial nematics (Brand and Pleiner 1981; Liu 1981), and several of the superfluid phases of ${ }^{3} \mathrm{He}$ (Brand et al. 1979; Brand and Pleiner 1981II, 1981III, 1982II; Pleiner and Brand 1983).

We close this Appendix by pointing out that the approaches using exclusively Poisson brackets, such as for example the investigations of Dzyaloshinskii and Volovick (1980) and by Edwards and Beris (Edwards and Beris 1998), do not contain reversible contributions from the memory matrix, since they focus exclusively on the instantaneous response.

Publisher's Note Springer Nature remains neutral with regard to jurisdictional claims in published maps and institutional affiliations.

\section{References}

Akopyan RS, Zeldovich BY (1984) Thermomechanical effects in deformed nematics. Sov Phys JETP 60:953-958

Aguilar Gutierrez OF, Rey AD (2014) Chiral graded structures in biological plywoods and in the beetle cuticle. Colloids and Interface Science Communications 3:18-22

Aguilar Gutierrez OF, Rey AD (2016) Theory and simulations of cholesteric film formation flows of dilute collagen solutions. Langmuir 32:11799-11812

Beens WW, de Jeu WH (1983) Flow measurements of the viscosity coefficients of two nematic liquid crystalline azoxybenzenes. J Physique (France) 44:129-136

Bouligand Y (1969) Sur l'existence de 'pseudomorphoses cholestériques' chez divers organismes vivants. J Physique (France) Colloques C4(30):90-103 
Bouligand Y (1972) Twisted fibrous arrangements in biological materials and cholesteric mesophases. Tissue Cell 4:189-217

Boychuk AN, Makarov DV, Zakhlevnykh (2016) Dynamics of liquid crystalline magnetic suspensions in a rotating magnetic field. Eur Phys J E 39:101

Brand H, Dörfle M, Graham R (1979) Hydrodynamic parameters and correlation functions of superfluid ${ }^{3} \mathrm{He}$. Ann Phys (N.Y.) 119:434-479

Brand H, Pleiner H (1981) Hydrodynamics of biaxial discotics. Phys Rev A 24:2477-2488

Brand H, Pleiner H (1981II) Linearized hydrodynamics of ${ }^{3} \mathrm{He}-\mathrm{A}$ in high magnetic fields. J Phys C 14:97-103

Brand H, Pleiner H (1981III) Broken symmetries and hydrodynamics of superfluid ${ }^{3} \mathrm{P}_{2}$ neutron star matter. Phys Rev D 24:3048-3057

Brand H, Pleiner H (1982) Number of elastic coefficients in a biaxial nematic liquid crystal. Phys Rev A 26:1783-1784

Brand H, Pleiner H (1982II) Linearized hydrodynamics of superfluid ${ }^{3} \mathrm{He}-\mathrm{A}_{1}$ : correlation functions and hydrodynamic parameters. $\mathrm{J}$ Phys (Paris) 43:369-380

Brand H, Pleiner H (1982III) Theory of flow alignment in biaxial nematics and nematic discotics. J Phys (Paris) 43:853-858

Brand HR (1986) Static effects of electric fields near the nematic and cholesteric to isotropic phase transitions. Mol Cryst Liq Cryst Lett 3:147-153

Brand HR (1986II) Dynamic effects of electric fields near the nematic and cholesteric to isotropic phase transitions. Mol Cryst Liq Cryst Lett 4:23-30

Brand HR, Pleiner H (1987) Nonlinear effects in the electrohydrodynamics of uniaxial nematic liquid crystals. Phys Rev A 35:3122-3127

Brand HR, Pleiner H (1988) New theoretical results for the Lehmann effect in cholesteric liquid crystals. Phys Rev A 37:2736-2738

Brand HR, Pleiner H, Ziebert F (2006) Macroscopic dynamics of polar nematic liquid crystals. Phys Rev E 74:021713

Brand HR, Pleiner H, Svenšek D (2011) Macroscopic behavior of systems with an axial dynamic preferred direction. Eur Phys J E 34:128

Brand HR, Pleiner H, Svenšek D (2013) Lehmann effects and rotatoelectricity in liquid crystalline systems made of achiral molecules. Phys Rev E 88:024501

Brand HR, Pleiner H, Svenšek D (2014) Reversible and dissipative macroscopic contributions to the stress tensor: active or passive? Eur Phys J E 37:83

Brand HR, Pleiner H (2017) On the influence of a network on optically isotropic fluid phases with tetrahedral/octupolar order. Eur Phys J E 40:34

Cladis PE, Couder Y, Brand HR (1985) Phase winding and flow alignment in freely suspended films of smectic C liquid crystals. Phys Rev Lett 55:2945-2948

Cladis PE, Finn PL, Brand HR (1995) Stable coexistence of spiral and target patterns in freely suspended films of smectic $\mathrm{C}$ liquid crystals. Phys Rev Lett 75:1518-1521

Cladis PE, Brand HR, Pleiner H (1999) Fluid biaxial banana smectics: Symmetry at work. Liq Cryst Today 9(3/4):1-6

de Gennes PG (1971) Short range order effects in the isotropic phase of nematic and cholesterics. Mol Cryst Liq Cryst 12:193-214

de Gennes PG (1975) The physics of liquid crystals. Clarendon Press, Oxford

de Groot SR, Mazur P (1962) Nonequilibrium Thermodynamics. North Holland, Amsterdam

Doi M (1981) Molecular dynamics and rheological properties of concentrated solutions or rodlike polymers in isotropic and liquid crystalline phases. J Polym Sci Pol Phys 19:229-243
Dzyaloshinskii IE, Volovick GE (1980) Poisson brackets in condensed matter physics. Ann Phys (N.Y.) 125:67-97

Edwards BJ, Beris AN (1998) Rotational motion and Poisson bracket structures in rigid particle systems and anisotropic fluid theory. Open Sys and Information Dyn 5:333-368

Ericksen JL (1960) A new constitutive theory for extrusion-extensional flow of anisotropic liquid crystalline polymer fluid. Arch Rat Mech Anal 4:231-237

Ericksen JL (1961) Conservation laws for liquid crystals. Trans Soc of Rheol 5:23-34

Figueiredo Neto AM, Galerne Y, Liébert L (1985) Characterization of a biaxial nematic lyophase studied by $\mathrm{x}$-ray diffraction and optical microscopy. J Phys Chem 89:3939-3941

Forster D, Lubensky TC, Martin PC, Swift J, Pershan PS (1971) Hydrodynamics of liquid crystals. Phys Rev Lett 26:1016-1019

Forster D (1974) Microscopic theory of flow alignment in nematic liquid crystals. Phys Rev Lett 32:1161-1164

Forster D (1974II) Hydrodynamics and correlation functions in ordered systems - nematic liquid crystals. Ann Phys (NY) 84:505534

Forster D (1975) Hydrodynamic fluctuations, broken symmetry, and correlation functions. W.A. Benjamin, Reading

Frank FC (1958) On the theory of liquid crystals. Discuss Faraday Soc 25:19-28

Grmela M, Öttinger HC (1997) Dynamics and thermodynamics of complex fluids. I. Development of a general formalism. Phys Rev E 56:6620-6632

Grmela M (2002) Lagrange hydrodynamics as extended Euler hydrodynamics: Hamiltonian and GENERIC structures. Phys Lett A 296:97-104

Hakobyan RS, Zeldovich BY, Seferyan H (2004) Thermomechanical coupling in a cylindrical hybrid-aligned nematic liquid crystal. J Exp Theor Phys 99:1039-1043

Jacobsen EA, Swift J (1982) Light scattering at the uniaxial - biaxial transition in nematic liquid crystals. Mol Cryst Liq Cryst 87:29-39

Jarkova E, Pleiner H, Müller HW, Fink A, Brand HR (2001) Hydrodynamics of nematic ferrofluids. Eur Phys J E 5:583-588

Jarkova E, Pleiner H, Müller HW, Brand HR (2003) Macroscopic dynamics of ferronematics. J Chem Phys 118:2422-2430

Kuzuu N, Doi M (1983) Constitutive equation for nematic liquid crystals under weak velocity gradient derived from a molecular kinetic equation. J Phys Soc Jap 52:3486-3494

Landau LD, Lifshitz EM (1959) Statistical physics. Butterworth, Oxford

Lehmann O (1900) Structur, System und magnetisches Verhalten flüssiger Krystalle und deren Mischbarkeit mit festen. Ann Phys (Leipzig) 2:649-705

Leslie FM (1968) Some thermal effects in cholesteric liquid crystals. Proc Roy Soc A 307:359-372

Leslie FM (1968II) Some constitutive equations for liquid crystals. Arch Rat Mech Anal 28:265-283

Leslie FM (1969) Continuum theory of cholesteric liquid crystals. Mol Cryst Liq Cryst 7:407-420

Liu M (1975) Hydrodynamics of ${ }^{3} \mathrm{He}$ near the $\boldsymbol{A}$ transition. Phys Rev Lett 35:1577-1580

Liu M (1981) Hydrodynamic theory of biaxial nematics. Phys Rev A 24:2720-2726

Livolant F, Bouligand Y (1978) New observations on the twisted arrangement of dinoflagellate chromosomes. Chromosoma 68:2144

Lubensky TC (1972) Hydrodynamics of cholesteric liquid crystals. Phys Rev A 6:452-470 
Lubensky TC (1973) Hydrodynamics of cholesterics in an external magnetic field. Mol Cryst Liq Cryst 23:99-109

Madhusudana NV, Pratibha R (1987) Electromechanical coupling in cholesteric liquid crystals. Mol Cryst Liq Cryst Lett 5:43-51

Madhusudana NV, Pratibha R (1989) An experimental investigation of electromechanical coupling in cholesteric liquid crystals. Liq Cryst 5:1827-1840

Martin PC, Parodi O, Pershan PS (1972) Unified hydrodynamic theory for crystals, liquid crystals and normal fluids. Phys Rev A 6:2401-2424

Mason WP (1958) Physical acoustics and the properties of solids. van Nostrand, Princeton

Melnick G, Saupe A (1987) Microscopic textures of micellar cholesteric liquid crystals. Mol Cryst Liq Cryst 145:95-110

Mertelj A, Lisjak D, Drofenik M, Čopič M (2013) Ferromagnetism in suspensions of magnetic platelets in liquid crystal. Nature 504:237-241

Mertelj A, Osterman N, Lisjak D, Čopič M (2014) Magneto-optic and converse magneto-electric effects in a ferromagnetic liquid crystal. Soft Matter 10:9065-9072

Miesowicz M (1935) Influence of a magnetic field on the viscosity of para-azoxyanisol. Nature (London) 136:261, 264

Miesowicz M (1946) The three coefficients of viscosity of anisotropic liquids. Nature (London) 158:27

Mori H (1965) Transport, collective motion, and Brownian motion. Prog Theor Phys 33:423-455

Murugesan YK, Rey AD (2010) Modeling textural processes during self-assembly of plant-based chiral nematic liquid crystals. Polymers 2:766-785

Öttinger HC, Grmela M (1997) Dynamics and thermodynamics of complex fluids. II. Illustration of a general formalism. Phys Rev E 56:6633-6655

Öttinger HC (2014) Irreversible dynamics, Onsager-Casimir symmetry, and an application to turbulence. Phys Rev E 90:042121

Oswald P, Dequidt A (2008) Direct measurement of the thermomechanical Lehmann coefficient in a compensated cholesteric liquid crystal. EPL 83:16005

Oswald P, Pieranski P (2005) Nematic and cholesteric liquid crystals. Taylor \& Francis, Boca Raton

Pleiner H, Brand H (1983) Linearized hydrodynamics of superfluid ${ }^{3} \mathrm{He}-\mathrm{B}$ in high magnetic fields. Phys Rev B 28:3782-3792

Pleiner (1984) Broken symmetries and hydrodynamics of hexatic B and smectic F and I liquid crystals. Mol Cryst Liq Cryst 114:103

Pleiner H, Brand H (1985) A new type of cholesteric liquid crystals. Phys Rev Lett 54:1817-1819

Pleiner H, Brand H (1987) Macroscopic description of compensated cholesteric and chiral smectic liquid crystals. Mol Cryst Liq Cryst Lett 5:61-65

Pleiner H, Brand H (1990) Frustrated states in chiral biaxial nematic liquid crystals. Mol Cryst Liq Cryst Lett 7:153-159

Pleiner H, Brand HR (1996). In: Buka A, Kramer L (eds) Pattern formation in liquid crystals. Springer, New York, pp 15-67

Pleiner H, Liu M, Brand HR (2002) Convective nonlinearities for the orientational tensor order parameter in polymeric systems. Rheol Acta 41:375-382
Pleiner H, Brand HR (2010) Comment on "Direct measurements of the thermomechanical Lehmann coefficient in a compensated cholesteric liquid crystal". EPL 89:26003

Pleiner H, Svenšek D, Brand HR (2013) Active polar two-fluid macroscopic dynamics. Eur Phys J E 36:135

Pleiner H, Brand HR (2014) Low symmetry tetrahedral nematic liquid crystal phases: Ambidextrous chirality and ambidextrous helicity. Eur Phys J 37:11

Pleiner H, Brand HR (2016) Tetrahedral order in liquid crystals. Braz J Phys 46:565-595

Pleiner H, Svenšek D, Brand HR (2016II) Hydrodynamics of Active Polar Systems in a (Visco)Elastic Background. Rheol Acta 55:857-870

Potisk $T$ et al (2017) Dynamic magneto-optic coupling in a ferromagnetic nematic liquid crystal. Phys Rev Lett 119:097802

Potisk $\mathrm{T}$ et al (2018) Magneto-optic dynamics in a ferromagnetic nematic liquid crystal. Phys Rev E 97:012701

Raikher YL, Stepanov VI (1996) Dynamic magneto-optical response of ferronematic liquid crystals. J Int Mat Sys Struc 7:550-554

Raikher YL, Stepanov VI (1999) Transient field-induced birefringence in a ferronematic. J Magn Magn Mat 201:182-185

Rey AD (2000) Theory of linear viscoelasticity of cholesteric liquid crystals. J Rheol 44:855-869

Rey AD (2010) Liquid crystal materials of biological materials and processes. Soft Matter 6:3402-3429

Rey AD, Herrera-Valencia EE (2012) Liquid crystal models of biological materials and silk spinning. Biopolymers 97:374396

Sahoo R, Rasna MV, Lisjak D, Mertelj A, Dhara S (2015) Magnetodielectric and magnetoviscosity response of a ferromagnetic liquid crystal at low magnetic fields. Appl Phys Lett 106:161905

Saupe A (1981) Elastic and flow properties of biaxial nematics. J Chem Phys 75:5118-51124

Sato S, Bono S, Tabe Y (2017) Unidirectional heat transport driven by rotating cholesteric droplets. J Phys Soc Jap 86:023601

Seki K, Ueda K, Okumura Y, Tabe Y (2011) Non-equilibrium dynamics of $2 \mathrm{D}$ liquid crystals driven by trans-membrane gas flow. J Phys: Condens Matter 23:284114

Svenšek D, Pleiner H, Brand HR (2006) Phase winding in chiral liquid crystalline monolayers due to Lehmann effects. Phys Rev Lett 96:140601

Svenšek D, Pleiner H, Brand HR (2008) Inverse Lehmann effects can be used as a microscopic pump. Phys Rev E 78:021703

Tabe Y, Yokoyama H (2003) Coherent collective precession of molecular rotors with chiral propellers. Nat Mater 2:806-809

Yamamoto T, Kuroda M, Sano M (2015) Three-dimensional analysis of thermo-mechanically rotating cholesteric liquid crystal droplets under a temperature gradient. EPL 109:46001

Yoshioka J, Ito F, Suzuki Y, Takahashi H, Takizawa H, Tabe Y (2014) Director/barycentric rotation in cholesteric droplets under temperature gradient. Soft Matter 10:5869-5877

Zwanzig R (1960) Ensemble method in the theory of irreversibility. J Chem Phys 33:1338-1341

Zwanzig R (1961) In Lectures in theoretical physics, vol.III. Interscience, New York 